\title{
Investigation into Factors Influencing Stereoselectivity in the Reactions of Heterocycles With Donor-Acceptor Substituted Rhodium Carbenoids
}

Simon J. Hedley, Dominic L. Ventura, Paulina M.Dominiak, Cara L. Nygren and Huw M. L. Davies*

Department of Chemistry, University at Buffalo, The State University of New York, Buffalo, New York 14260-3000.

hdavies@buffalo.edu

\section{Supporting Information}

Table Of Contents

General Considerations

$\mathrm{S} 2$

Experimental Procedures And Characterization Data

S3-S26

${ }^{1} \mathrm{H}$ NOE Interactions for dienes 10, 11, 17a and $\mathbf{1 7 b}$

S27

${ }^{1} \mathrm{H}$ NMR Spectrum of $\mathbf{1 0}$

S28

${ }^{1} \mathrm{H}$ NMR Spectrum of $\mathbf{1 7 a}$

S29

${ }^{1} \mathrm{H}$ NMR Spectrum of $\mathbf{2 3}$

S30

${ }^{1} \mathrm{H}$ NMR Spectrum of $\mathbf{2 6}$

S31

${ }^{1} \mathrm{H}$ NMR Spectrum of 27

S32

${ }^{1} \mathrm{H}$ NMR Spectrum of $\mathbf{3 2}$

S33

${ }^{1} \mathrm{H}$ NMR Spectrum of 34

S34

${ }^{1} \mathrm{H}$ NMR Spectrum of $\mathbf{3 5}$

S35

${ }^{1} \mathrm{H}$ NMR Spectrum of $\mathbf{3 7}$

S36

X-ray Structure of 5

S37

$\mathrm{X}$-ray Structure of 9

S38

X-ray Structure of ent-12

S39

$\mathrm{X}$-ray Structure of $\mathbf{1 6}$

S40

$\mathrm{X}$-ray Structure of $\mathbf{2 0}$

S41

$\mathrm{X}$-ray Structure of $\mathbf{3 2}$

S42

Circular Dichroism Spectra of 20

Circular Dichroism Spectra of 21 


\section{General Considerations}

All reactions were conducted in oven or flame-dried glassware under an inert atmosphere of dry argon. All reagents were used as received from commercial suppliers unless otherwise stated. Dichloromethane, hexane and tetrahydrofuran were purified by passage through a bed of activated alumina. 2,2-Dimethylbutane was distilled from sodium metal, and then degassed by bubbling argon through the solvent for $15 \mathrm{~min}$ prior to use. $N$-Boc Pyrrole 3 was purified by bulb-to-bulb kugelrohr distillation immediately before use. Furan 7 was purified by distillation from potassium hydroxide. Flash chromatography was performed on silica gel (32-63D $60 \AA$ ) according to the method of W.C. Still. ${ }^{1}$ Thin layer chromatography (TLC) was performed on aluminium backed plates pre-coated with silica (0.25 mm, 60F-254) which were developed using standard visualizing agents: UV fluorescence (254 and $366 \mathrm{~nm}$ ), phosphomolybdic acid / $\Delta$. Melting points were determined using an electrothermal melting point apparatus and are uncorrected. Specific optical rotations $\left([\alpha]_{\mathrm{D}}\right)$ were measured on a digital polarimeter at $25{ }^{\circ} \mathrm{C} .{ }^{1} \mathrm{H}$ NMR spectra were recorded on a Nuclear Magnetic Resonance spectrometer at 500, 400, or $300 \mathrm{MHz}$, and ${ }^{13} \mathrm{C}$ NMR spectra were recorded on a Nuclear Magnetic Resonance spectrometer at $125 \mathrm{MHz}$ or $75 \mathrm{MHz}$, calibrated by using residual undeuterated solvent as an internal standard. The following abbreviations apply: (b) broad. (s) singlet, (d) doublet, (t) triplet, (q) quartet, (m) multiplet, (dd) double doublet, etc. Chemical shifts are given in ppm. $J$ values are recorded in $\mathrm{Hz}$ and are rounded to the nearest half-integer. Infrared (IR) spectra were determined using a FTIR spectrometer. Low resolution mass spectra $(\mathrm{m} / \mathrm{z})$ and high resolution mass spectra (HRMS) were obtained from an external service provider. Elemental analyses were determined by an external service provider. Diastereomeric ratios were determined by values derived from the $500 \mathrm{MHz}{ }^{1} \mathrm{H} \mathrm{NMR}$ spectra of the crude reaction mixture. Enantiomeric excess was determined by high performance liquid chromatography (HPLC) using chiral analytical columns with 2propanol in hexane as eluant.

The known compounds methyl para-bromophenyldiazoacetate $4,{ }^{2} \mathrm{Rh}_{2}(S \text {-DOSP })_{4} 2$ and $\mathrm{Rh}_{2}(R \text {-DOSP })_{4},{ }^{3}$ tert-butyl 3-methyl-1H-indole-1-carboxylate $\mathbf{2 4},{ }^{4}$ tert-butyl 2-methyl-

\footnotetext{
${ }^{1}$ Still, W. C.; Kahn, M.; Mitra, M. J. Org. Chem. 1978, 43, 2923.

${ }^{2}$ Davies, H. M. L.; Hansen, T.; Churchill, M. R. J. Am. Chem. Soc. 2000, 122, 3063.
} 
$1 H$-indole-1-carboxylate $\mathbf{2 5},{ }^{4}$ tert-butyl 3 -(hydroxymethyl)-1 $H$-indole-1-carboxylate, ${ }^{5}$ methyl phenyldiazoacetate $\mathbf{2 9},{ }^{6} \quad \mathrm{Rh}_{2}(S \text {-PTTL })_{4}{ }^{7}(S)$-ethyl 2-diazo-2-(2phenylacetoxy)propanoate $\mathbf{3 3}^{8}$ and tert-butyl 5-bromo-3-formyl-1 $H$-indole-1carboxylate $^{9}$ were prepared by literature procedures and the spectral data showed good agreement with the literature data.

\section{Experimental Procedures and Characterization Data}

\section{Compound (5)}

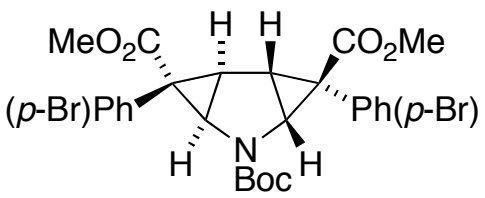

Procedure A: A solution of methyl para-bromophenyldiazoacetate 4 (255 mg, 1.00 mmol, 1.00 eq.) in hexanes $(7.5 \mathrm{~mL})$ was added by syringe pump over $1 \mathrm{~h}$ to a solution of $N$-Boc pyrrole 3 (1.00 mL, $5.98 \mathrm{mmol}, 5.98$ eq.) and $\mathrm{Rh}_{2}(S \text {-DOSP })_{4}(19 \mathrm{mg}, 0.01 \mathrm{mmol}$, 0.01 eq.) in hexanes $(5 \mathrm{~mL})$. The reaction mixture was stirred for an additional $1 \mathrm{~h}$, and then concentrated in vacuo. Purified by flash chromatography on silica gel using 9:1 to 5:1 hexane/ $\mathrm{Et}_{2} \mathrm{O}$ as eluant to isolate a white solid, $238 \mathrm{mg}(77 \%)$.

Procedure B: A solution of methyl para-bromophenyldiazoacetate 4 (457 mg, 1.79 mmol, 3.00 eq.) in hexanes $(10 \mathrm{~mL})$ was added by syringe pump over $1 \mathrm{~h}$ to a solution of $N$-Boc pyrrole 3 ( $0.10 \mathrm{~mL}, 0.61 \mathrm{mmol}, 1.00$ eq.) and $\mathrm{Rh}_{2}(S \text {-DOSP })_{4}(34 \mathrm{mg}, 0.018 \mathrm{mmol}$, 0.01 eq.) in hexanes $(5 \mathrm{~mL})$. The reaction mixture was stirred for an additional $1 \mathrm{~h}$, and then concentrated in vacuo. Purified by flash chromatography on silica gel using 9:1 to 5:1 hexane/ $\mathrm{Et}_{2} \mathrm{O}$ as eluant to isolate a white solid, $354 \mathrm{mg}(93 \%)$.

Procedure C: A solution of methyl para-bromophenyldiazoacetate 4 (10.5 mg, 0.04 mmol, 0.5 eq. $)$ in hexanes $(2 \mathrm{~mL})$ was added by syringe pump over $1 \mathrm{~h}$ to a solution of racemic 6 (32 mg, $0.08 \mathrm{mmol}, 1.00$ eq.) and $\mathrm{Rh}_{2}(S \text {-DOSP })_{4}(2 \mathrm{mg}, 0.001 \mathrm{mmol}, 0.02$ eq. $)$ in hexanes $(2 \mathrm{~mL})$ under reflux. The reaction mixture was stirred under reflux for an

\footnotetext{
${ }^{3}$ Davies, H. M. L.; Bruzinski, P. R.; Lake, D. H.; Kong, N.; Fall, M. J. J. Am. Chem. Soc. 1996, 118, 6897.

${ }^{4}$ Clark, R. D.; Muchowski, J. M.; Fisher, L. E.; Flippin, L. A.; Repke, D. B., Souchet, M. Synthesis 1991, 871.

${ }^{5}$ Oliveira, D.; Coelho, F. Synth. Commun. 2000, 30, 2143.

${ }^{6}$ Takamura, N.; Mizoguchi, T.; Koga, K.; Yamada, S. Tetrahedron 1975, 31, 227.

${ }^{7}$ Hashimoto, S.; Watanabe, N.; Sato, T.; Shiro, M.; Ikegami, S. Tetrahedron Lett. 1993, 34, 5109.

${ }^{8}$ Davies, H. M. L.; Hedley, S. J.; Bohall, B. R. J. Org. Chem. 2005, 70, 10737.

${ }^{9}$ Wang, W.; Xiong, C.; Zhang, J.; Hruby, V. J. Tetrahedron 2002, 58, 3101.
} 
additional $1 \mathrm{~h}$, and allowed to cool to room temperature and then concentrated in vacuo. Purified by flash chromatography on silica gel using 9:1 to $5: 1$ hexane/ $\mathrm{Et}_{2} \mathrm{O}$ as eluant to isolate a white solid, $12.5 \mathrm{mg}(49 \%)$.

Mp 196-199 ${ }^{\circ}$ C. [_]D 234.4 (83\% ee) (c 1.00, $\left.\mathrm{CHCl}_{3}\right) .{ }^{1} \mathrm{H}$ NMR (300 $\left.\mathrm{MHz}, \mathrm{CDCl}_{3}\right)$ : _7.55-7.48 (m, 4H), $7.16(\mathrm{~d}, 2 \mathrm{H}, J=8.5 \mathrm{~Hz}), 7.11$ (d, 2H, J=8.0 Hz), 3.56 (s, 3H), 3.52 (s, $3 \mathrm{H}), 3.07$ (d, 1H, $J=6.5 \mathrm{~Hz}), 2.98$ (d, 1H, J=6.5 Hz), 2.59 (d, 1H, J=6.5 Hz), 2.56 (d, 1H, $J=6.5 \mathrm{~Hz}), 1.51(\mathrm{~s}, 9 \mathrm{H}) .{ }^{13} \mathrm{C} \mathrm{NMR}\left(75 \mathrm{MHz}, \mathrm{CDCl}_{3}\right)$ : $171.1,170.9,154.3,133.4,133.0$, 132.0, 131.8, 130.9, 130.7, 122.2, 122.1, 81.5, 52.7, 52.6, 47.7, 47.1, 37.0, 36.8, 32.9, 31.7, 28.4. IR $\left(\mathrm{CHCl}_{3}\right): 2973,1714,1699,1409,1346,1324,1237,1128,965,868,739$, $703 \mathrm{~cm}^{-1} . m / z$ (EI): $623\left(\mathrm{M}^{81} \mathrm{Br}_{2} \mathrm{Na}^{+}, 33 \%\right), 621\left(\mathrm{M}^{81} \mathrm{Br}^{79} \mathrm{BrNa}^{+}, 65 \%\right), 619\left(\mathrm{M}^{79} \mathrm{Br}_{2} \mathrm{Na}^{+}\right.$, 32\%), 591 (55\%), 589 (100\%), 587 (48\%). Anal. Calcd. for $\mathrm{C}_{27} \mathrm{H}_{27} \mathrm{Br}_{2} \mathrm{NO}_{6}: \mathrm{C}, 52.19 ; \mathrm{H}$, 4.38; N, 2.25. Found: C, 51.87; H, 4.14; N, 2.25. HPLC analysis: 83\% ee (Chiralcel AD$\mathrm{H}, 1.0 \% i-\mathrm{PrOH}$ in hexane, $0.8 \mathrm{ml} / \mathrm{min},{ }_{-}=254 \mathrm{~nm}, \mathrm{t}_{\mathrm{R}}=32.1 \mathrm{~min}$, minor; $\mathrm{t}_{\mathrm{R}}=47.5 \mathrm{~min}$, major).

$(1 R, 5 R, 6 S)-2-t e r t-b u t y l$ 6-methyl 6-(4-bromophenyl)-2-aza-bicyclo[3.1.0]hex-3-ene2,6-dicarboxylate (6)

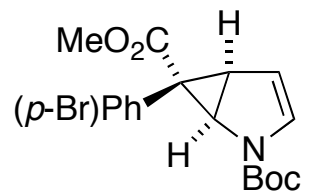

A solution of methyl para-bromophenyldiazoacetate 4 (1.00 g, $3.92 \mathrm{mmol}, 1.00$ eq.) in $N$-Boc pyrrole $3(8 \mathrm{~mL})$ was added by syringe pump over $1 \mathrm{~h}$ to a solution of $\mathrm{Rh}_{2}(S$ $\mathrm{DOSP}_{4}(37 \mathrm{mg}, 0.02 \mathrm{mmol}, 0.005$ eq.) in $N$-Boc pyrrole $3(7 \mathrm{~mL})$. The reaction mixture was stirred for an additional $1 \mathrm{~h}$, and then concentrated in vacuo. Purified by flash chromatography on silica gel using 9:1 hexane/ $\mathrm{Et}_{2} \mathrm{O}$ as eluant to isolate $\mathbf{6}$ as a white solid, $832 \mathrm{mg}(54 \%)$ and 5 as a white solid, $414 \mathrm{mg}(34 \%)$. Mp $132-134{ }^{\circ} \mathrm{C}$. $[\alpha]_{\mathrm{D}} 337.4(85 \%$ ee) $\left(c\right.$ 1.00, $\left.\mathrm{CHCl}_{3}\right) .{ }^{1} \mathrm{H} \mathrm{NMR}\left(400 \mathrm{MHz}, \mathrm{CDCl}_{3}\right): \delta 7.37(\mathrm{~d}, 2 \mathrm{H}, J=8.0 \mathrm{~Hz}), 7.03-6.95$ (m, 2H), $6.15(\mathrm{~d}, 0.41 \mathrm{H}, J=3.0 \mathrm{~Hz}$, minor rotamer), $6.00(\mathrm{~d}, 0.59 \mathrm{H}, J=3.5 \mathrm{~Hz})$, major rotamer), 5.21-5.16 (m, 0.48 H, minor rotamer), 5.13-5.08 (m, 0.52 H, major rotamer), 4.69 (d, $0.57 \mathrm{H}, J=6.5 \mathrm{~Hz}$, major rotamer), 4.59 (d, $0.43 \mathrm{H}, J=7.0 \mathrm{~Hz}$, minor rotamer), $3,61(\mathrm{~s}, 1.23 \mathrm{H}$, minor rotamer), $3.58(\mathrm{~s}, 1.77 \mathrm{H}$, major rotamer), 3.31-3.29 $(\mathrm{m}, 1 \mathrm{H}), 1.58$ (s, $3.52 \mathrm{H}$, minor rotamer), $1.45(\mathrm{~s}, 5.48 \mathrm{H}$, major rotamer). Due to the presence of 
rotamers arising from the Boc group, the ${ }^{13} \mathrm{C}$ NMR spectrum was complicated, and resonances are not included here. IR $\left(\mathrm{CHCl}_{3}\right): 3112,2986,1701,1582,1393,1357,1244$, 1177, 1139, 963, 765, $735 \mathrm{~cm}^{-1}$. HRMS (EI) Calcd. for $\mathrm{C}_{18} \mathrm{H}_{20} \mathrm{O}_{4} \mathrm{~N}^{79} \mathrm{Br}$ : 393.0570. Found: 393.0570. HRMS (EI) Calcd. for $\mathrm{C}_{18} \mathrm{H}_{20} \mathrm{O}_{4} \mathrm{~N}^{81} \mathrm{Br}$ : 395.0550. Found: 395.0546. Anal. Calcd. for $\mathrm{C}_{18} \mathrm{H}_{20} \mathrm{O}_{4} \mathrm{NBr}$ : C, 54.84; H, 5.11; N, 3.55. Found: C, 55.07; H, 5.27; N, 3.55. HPLC analysis: $85 \%$ ee (Chiralcell AD-H, $0.5 \% i-\mathrm{PrOH}$ in hexane, $0.8 \mathrm{ml} / \mathrm{min},-=254$ $\mathrm{nm}, \mathrm{t}_{\mathrm{R}}=18.9$ min, minor; $\mathrm{t}_{\mathrm{R}}=21.2 \mathrm{~min}$, major).

(1S,5S,6R)-Methyl 6-(4-bromophenyl)-2-oxa-bicyclo[3.1.0]hex-3-ene-6-carboxylate (8), Compound (9), (2E,4Z)-Methyl 2-(4-bromophenyl)-6-oxohexa-2,4-dienoate (10) and Methyl 2-(4-bromophenyl)-3-((1Z,3E)-4-(4-bromophenyl)-5-methoxy-5oxopenta-1,3-dienyl)oxirane-2-carboxylate (11)
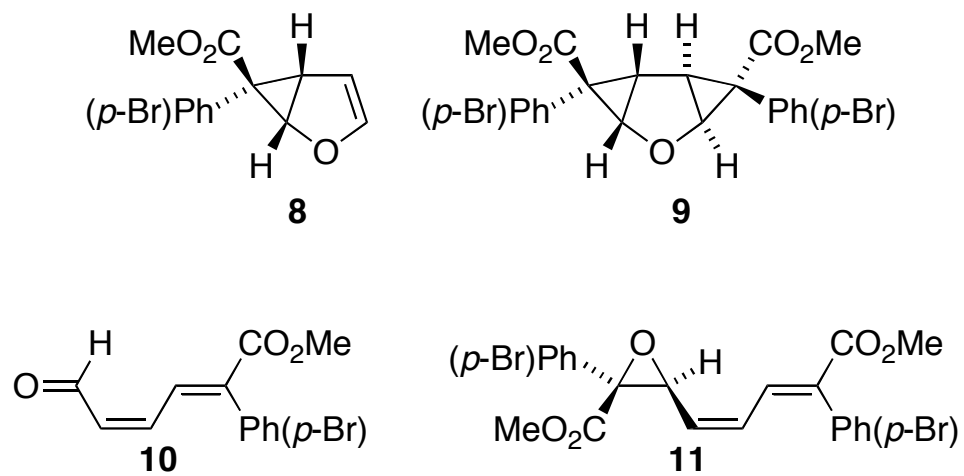

Procedure A: A solution of methyl para-bromophenyldiazoacetate 4 (255 mg, 1.00 mmol, 1.00 eq.) in hexanes $(7.5 \mathrm{~mL})$ was added by syringe pump over $1 \mathrm{~h}$ to a solution of furan 7 (0.5 mL, $6.87 \mathrm{mmol}, 6.87$ eq.) and $\mathrm{Rh}_{2}(S \text {-DOSP })_{4}(19 \mathrm{mg}, 0.01 \mathrm{mmol}, 0.01$ eq. $)$ in hexane $(5 \mathrm{~mL})$. The reaction mixture was stirred for an additional $1 \mathrm{~h}$, and then concentrated in vacuo. Purified by flash chromatography on silica gel using 9:1 to 5:1 hexane/ $\mathrm{Et}_{2} \mathrm{O}$ as eluant to isolate $\mathbf{8}$ as a white solid, $180 \mathrm{mg}(61 \%) ; \mathbf{1 0}$ as a yellow solid, 7 $\mathrm{mg}(2 \%)$; and $\mathbf{9}$ as a white solid, $83 \mathrm{mg}(8 \%)$.

Procedure B: A solution of methyl para-bromophenyldiazoacetate 4 (255 mg, 1.00 mmol, 1.00 eq.) in furan 7 ( $8 \mathrm{~mL}$ ) was added by syringe pump over $2 \mathrm{~h}$ to a solution of $\mathrm{Rh}_{2}(S \text {-DOSP })_{4}(19 \mathrm{mg}, 0.01 \mathrm{mmol}, 0.01$ eq. $)$ in furan $9(5 \mathrm{~mL})$. The reaction mixture was stirred for an additional $1 \mathrm{~h}$, and then concentrated in vacuo. Purified by flash 
chromatography on silica gel using 9:1 hexane/Et $2 \mathrm{O}$ as eluant to isolate $\mathbf{8}$ as a white solid, $190 \mathrm{mg}$ (65\%); and $\mathbf{1 0}$ as a pale yellow solid, $83 \mathrm{mg}(28 \%)$.

Procedure C: A solution of methyl para-bromophenyldiazoacetate 4 (471 mg, 1.85 mmol, 2.5 eq.) in hexanes $(10 \mathrm{~mL})$ was added by syringe pump over $1 \mathrm{~h}$ to a solution of furan 7 (0.067 mL, $0.92 \mathrm{mmol}, 1$ eq.) and $\mathrm{Rh}_{2}(S \text {-DOSP })_{4}(19 \mathrm{mg}, 0.01 \mathrm{mmol}, 0.01$ eq. $)$ in hexane $(5 \mathrm{~mL})$. The reaction mixture was stirred for an additional $1 \mathrm{~h}$, and then concentrated in vacuo. Purified by flash chromatography on silica gel using 9:1 to 5:1 hexane/Et ${ }_{2} \mathrm{O}$ as eluant to isolate $\mathbf{8}$ as a white solid, $14 \mathrm{mg}(5 \%) ; \mathbf{1 1}$ as a pale yellow solid, $32 \mathrm{mg}(12 \%)$; and 9 as a white solid, $328 \mathrm{mg}(68 \%)$.

Procedure D: A solution of methyl para-bromophenyldiazoacetate 4 (18 mg, $7 \mu \mathrm{mol}$, 0.5 eq.) in hexanes $(2 \mathrm{~mL})$ was added by syringe pump over $1 \mathrm{~h}$ to a solution of racemic 8 (41 mL, $0.14 \mathrm{mmol}, 1.0$ eq.) and $\mathrm{Rh}_{2}(S \text {-DOSP })_{4}(1.3 \mathrm{mg}, 0.07 \mu \mathrm{mol}, 0.01 \mathrm{eq}$.) in hexane $(5 \mathrm{~mL})$ under reflux. The reaction mixture was stirred at reflux for an additional $30 \mathrm{~min}$, and then allowed to cool top room temperature and concentrated in vacuo. Purified by flash chromatography on silica gel using 9:1 hexane/ $\mathrm{Et}_{2} \mathrm{O}$ as eluant to isolate 9 as a white solid, $18 \mathrm{mg}(48 \%)$.

Compound 8: $\mathrm{Mp} 85-88{ }^{\circ} \mathrm{C} .[\alpha]_{\mathrm{D}}-76.0(93 \%$ ee $)\left(c 1.09, \mathrm{CHCl}_{3}\right) .{ }^{1} \mathrm{H}$ NMR $(300 \mathrm{MHz}$, $\left.\mathrm{CDCl}_{3}\right): \_7.41(\mathrm{~d}, 2 \mathrm{H}, J=8.5 \mathrm{~Hz}), 7.06(\mathrm{~d}, 2 \mathrm{H}, J=8.5 \mathrm{~Hz}), 5.95(\mathrm{~d}, 1 \mathrm{H}, J=2.5 \mathrm{~Hz}), 5.23(\mathrm{t}$, $1 \mathrm{H}, J=2.5 \mathrm{~Hz}), 5.13(\mathrm{~d}, 1 \mathrm{H}, J=5.5 \mathrm{~Hz}), 3.64(\mathrm{~s}, 3 \mathrm{H}), 3.31(\mathrm{dd}, 1 \mathrm{H}, J=5.5,2.5 \mathrm{~Hz}) .{ }^{13} \mathrm{C}$ NMR (75 MHz, $\left.\mathrm{CDCl}_{3}\right)$ :_173.3, 147.5, 134.4, 131.1, 129.8, 121.4, 103.8, 70.7, 52.5, 39.3, 27.2. IR ( $\left.\mathrm{CHCl}_{3}\right): 2951,2677,2357,2257,1697,1585,1490,1436,1314,1258$, 1144, 1071, 1056, 985, $905 \mathrm{~cm}^{-1} \cdot \mathrm{m} / \mathrm{z}$ (EI): $296\left(\mathrm{M}^{81} \mathrm{Br}^{+}, 25 \%\right), 294\left(\mathrm{M}^{79} \mathrm{Br}^{+}, 25 \%\right), 237$ (97\%), 235 (100\%), 128 (65\%). Anal. Calcd. for $\mathrm{C}_{13} \mathrm{H}_{11} \mathrm{BrO}_{3}: \mathrm{C}, 52.91 ; \mathrm{H}, 3.76$, found $\mathrm{C}$, 
53.01; H, 3.74. HPLC analysis: 93\% ee (Chiralcel OD-H 1\% $i$-PrOH in hexane, 1.0 $\mathrm{ml} / \mathrm{min},{ }_{-}=254 \mathrm{~nm}, \mathrm{t}_{\mathrm{R}}=8.1 \mathrm{~min}$, major; $\mathrm{t}_{\mathrm{R}}=9.1 \mathrm{~min}$, minor).

Compound 9: mp 180-183 ${ }^{\circ} \mathrm{C}$. [_] $5.5(96 \%$ ee $)\left(c 1.00 \mathrm{CHCl}_{3}\right) .{ }^{1} \mathrm{H}$ NMR $(300 \mathrm{MHz}$, $\left.\mathrm{CDCl}_{3}\right): \_7.54(\mathrm{~d}, 4 \mathrm{H}, J=8.5 \mathrm{~Hz}), 7.20(\mathrm{~d}, 4 \mathrm{H}, J=8.5 \mathrm{~Hz}), 3.51$ (s, 6H), $3.40(\mathrm{~d}, 2 \mathrm{H}, J=5.5$ $\mathrm{Hz}), 2.47(\mathrm{~d}, 2 \mathrm{H}, J=5.5 \mathrm{~Hz}) .{ }^{13} \mathrm{C}$ NMR $\left(75 \mathrm{MHz}, \mathrm{CDCl}_{3}\right): \_170.9,133.5,131.7,130.5$, 122.1, 67.9, 52.6, 36.5, 34.1. IR $\left(\mathrm{CHCl}_{3}\right): 2949,1712,1489,1434,1323,1250,1070$, 1011, $970 \mathrm{~cm}^{-1} . \quad m / z$ (ESI): $525\left(\mathrm{M}^{81} \mathrm{Br}_{2} \mathrm{H}^{+}, 37 \%\right), 523\left(\mathrm{M}^{81} \mathrm{Br}^{79} \mathrm{BrH}^{+}, 60 \%\right), 521$ $\left(\mathrm{M}^{79} \mathrm{Br}_{2} \mathrm{H}^{+}, 32 \%\right), 277$ (24\%). Anal. Calcd. for $\mathrm{C}_{22} \mathrm{H}_{18} \mathrm{Br}_{2} \mathrm{O}_{5}: \mathrm{C}, 50.60 ; \mathrm{H}, 3.47$. Found: $\mathrm{C}$, 50.31, H, 3.46. HPLC analysis: 96\%ee (Chiralcel AD-H, $0.8 \% i$-PrOH in hexane, 0.8 $\mathrm{ml} / \mathrm{min},{ }_{-}=254 \mathrm{~nm}, \mathrm{t}_{\mathrm{R}}=33.7 \mathrm{~min}$, minor; $\mathrm{t}_{\mathrm{R}}=44.6 \mathrm{~min}$, major).

Compound 10: $\mathrm{Mp} 150-152{ }^{\circ} \mathrm{C} .{ }^{1} \mathrm{H}$ NMR (400 MHz, $\left.\mathrm{CDCl}_{3}\right)$ : $\delta 9.56$ (d, $1 \mathrm{H}, J=8.0 \mathrm{~Hz}$ ), 7.72-7.51 (m, 3H), 7.15 (d, 2H, $J=8.5 \mathrm{~Hz}), 7.07$ (dd, 1H, $J=15.0,11.5 \mathrm{~Hz}), 6.47$ (dd, $1 \mathrm{H}$, $J=15.0,8.0 \mathrm{~Hz}), 3.84$ (s, 3H). ${ }^{13} \mathrm{C}$ NMR $\left(75 \mathrm{MHz}, \mathrm{CDCl}_{3}\right): \delta 192.9,166.2,145.1,139.6$, 137.4, 136.6, 132.5, 131.7, 131.6, 123.3, 52.7. IR $\left(\mathrm{CHCl}_{3}\right): 2953,1701,1674,1437$, 1279, 1219, 1147, 1115, 974, $833 \mathrm{~cm}^{-1} \cdot m / z$ (EI): $296\left(\mathrm{M}^{81} \mathrm{Br}^{+}, 9 \%\right), 294\left(\mathrm{M}^{79} \mathrm{Br}^{+}, 10 \%\right)$, 267 (59\%), 265 (62\%), 237 (80\%), 235 (84\%), 128 (100\%). HRMS (EI) Calcd. for $\mathrm{C}_{13} \mathrm{H}_{11} \mathrm{O}_{3}{ }^{79} \mathrm{Br}$ : 293.9886. Found: 293.9884. HRMS (EI) Calcd. for $\mathrm{C}_{13} \mathrm{H}_{11} \mathrm{O}_{3}{ }^{81} \mathrm{Br}$ : 295.9866. Found: 295.9868. Relative stereochemistry was assigned by NOE data (see page S27 for major interactions).

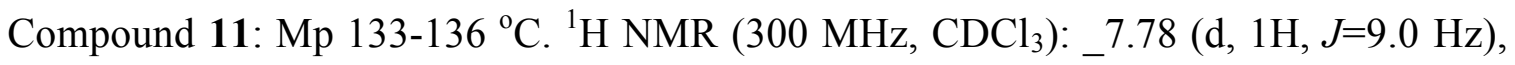
$7.55(\mathrm{~m}, 6 \mathrm{H}), 7.08$ (d, 2H, J=8.0 Hz), $6.35(\mathrm{dd}, 1 \mathrm{H}, J=12.0,11.5 \mathrm{~Hz}), 5.56(\mathrm{dd}, 1 \mathrm{H}$, $J=10.5,9.0 \mathrm{~Hz}), 4.00(\mathrm{~d}, 1 \mathrm{H}, J=9.0 \mathrm{~Hz}), 3.79$ (s, 3H), $3.76(\mathrm{~s}, 3 \mathrm{H}) .{ }^{13} \mathrm{C} \mathrm{NMR}(75 \mathrm{MHz}$, $\left.\mathrm{CDCl}_{3}\right): \_167.3,166.9,135.0,133.3,133.1,132.9,131.8,131.7,131.7,131.3,130.8$ 
128.3, 123.2, 122.5, 64.8, 61.3, 52.9, 52.5. IR $\left(\mathrm{CHCl}_{3}\right): 2952,2919,2849,1737,1712$, $1487,1435,1242,1198,1173,1098,1071,1010 \mathrm{~cm}^{-1} \cdot m / z(E I): 524\left(\mathrm{M}^{81} \mathrm{Br}_{2}^{+}, 37 \%\right), 522$ $\left(\mathrm{M}^{81} \mathrm{Br}^{79} \mathrm{Br}^{+}, 69 \%\right), 520\left(\mathrm{M}^{79} \mathrm{Br}_{2}^{+}, 37 \%\right), 490$ (41\%), 458 (40\%), 430 (71\%), 351 (65\%), $322(61 \%), 295$ (68\%), 252 (75\%), 215 (100\%). Anal. Calcd. for $\mathrm{C}_{22} \mathrm{H}_{18} \mathrm{Br}_{2} \mathrm{O}_{5}$ : C, 50.60;

H, 3.47. Found C, 50.79, H, 3.62. Relative stereochemistry was assigned by NOE data (see page S27 for major interactions).

(1S,5S,6R)-Methyl 6-(4-bromophenyl)-2-oxa-bicyclo[3.1.0]hexane-6-carboxylate (12)

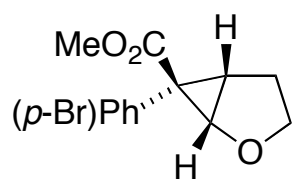

12

A Parr ${ }^{\mathrm{TM}}$ flask was charged with a solution of $\mathbf{8}(20 \mathrm{mg}, 68 \mu \mathrm{mol}, 1.00$ eq.) in ethanol (10 $\mathrm{mL})$ and $\mathrm{RhCl}\left(\mathrm{PPh}_{3}\right)_{3}(5 \mathrm{mg}, 0.7 \mu \mathrm{mol}, 0.01$ eq. $)$. The reaction vessel was placed on a Parr $^{\mathrm{TM}}$ hydrogenation apparatus, and evacuated and repressurized with hydrogen gas at 50 psi. The reaction mixture was shaken at 50 psi for $24 \mathrm{~h}$, and then filtered through Celite and concentrated in vacuo. The residue was purified by flash chromatography on silica gel using 9:1 hexane/ $\mathrm{Et}_{2} \mathrm{O}$ as eluant. Isolated $17 \mathrm{mg}(82 \%)$ of product, which showed comparitive spectral data to ent-12 (see below), except that chiral HPLC showed the opposite major enantiomer.

$(1 R, 5 R, 6 S)$-Methyl 6-(4-bromophenyl)-2-oxa-bicyclo[3.1.0]hexane-6-carboxylate (ent-12)

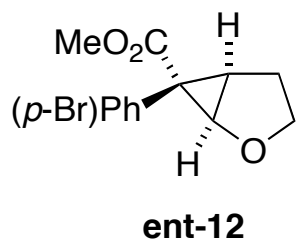

A solution of methyl para-bromophenyldiazoacetate 4 (82.4 mg, $0.32 \mathrm{mmol}, 1.3 \mathrm{eq}$.$) in$ hexanes $(10 \mathrm{~mL})$ was added by syringe pump over $1 \mathrm{~h}$ to a solution of 2,3-dihydrofuran 
$13(0.020 \mathrm{~mL}, 0.26 \mathrm{mmol}, 1$ eq. $)$ and $\mathrm{Rh}_{2}(S \text {-DOSP })_{4}(5 \mathrm{mg}, 0.0026 \mathrm{mmol}, 0.01$ eq. $)$ in hexane $(5 \mathrm{~mL})$. The reaction mixture was stirred for an additional $1 \mathrm{~h}$, and then concentrated in vacuo. Purified by flash chromatography on silica gel using 9:1 hexane/ $\mathrm{Et}_{2} \mathrm{O}$ as eluant to isolate a white solid, $62 \mathrm{mg}(81 \%)$. Mp $97-100{ }^{\circ} \mathrm{C}$. [ $]_{\mathrm{D}}-14.0$ (77\% ee) $\left(c\right.$ 1.00, $\left.\mathrm{CHCl}_{3}\right) .{ }^{1} \mathrm{H}$ NMR $\left(300 \mathrm{MHz}, \mathrm{CDCl}_{3}\right)$ :_7.52 (d, $\left.2 \mathrm{H}, J=8.5 \mathrm{~Hz}\right), 7.21$ (d, $2 \mathrm{H}, J=8.5 \mathrm{~Hz}), 4.50$ (d, 1H, $J=6.0 \mathrm{~Hz}), 3.71(\mathrm{~m}, 1 \mathrm{H}), 3.57$ (s, 3H), 2.69 (t, $1 \mathrm{H}, J=6.0$ $\mathrm{Hz}), 2.51(\mathrm{q}, 1 \mathrm{H}, J=8.5 \mathrm{~Hz}), 2.31(\mathrm{~m}, 1 \mathrm{H}), 1.85(\mathrm{~m}, 1 \mathrm{H}) .{ }^{13} \mathrm{C} \mathrm{NMR}\left(75 \mathrm{MHz}, \mathrm{CDCl}_{3}\right)$ : _171.2, 133.12, $131.6(2 \mathrm{C}), 131.2,121.7,70.2,69.8,52.2,37.5,32.4,26.0 . \quad$ IR $\left(\mathrm{CHCl}_{3}\right)$ : $2950,1705,1588,1489,1435,1397,1327,1248,1091,1070,1034,1012 \mathrm{~cm}^{-1} . m / z(E I)$ : $298\left(\mathrm{M}^{81} \mathrm{Br}^{+}, 22 \%\right), 296\left(\mathrm{M}^{79} \mathrm{Br}^{+}, 22 \%\right), 283$ (18\%), 281 (17\%), 238 (21\%), 209 (19\%), 183 (23\%), 157 (18\%), 129 (100\%). Anal. Calcd. for $\mathrm{C}_{13} \mathrm{H}_{13} \mathrm{BrO}_{3}$ : C, 52.55; H, 4.41 . Found: $\mathrm{C}, 52.41 ; \mathrm{H}, 4.50$. HPLC analysis: $77 \%$ ee (Chiralcel OD-H, $0.5 \% i-\mathrm{PrOH}$ in hexane, $1.0 \mathrm{ml} / \mathrm{min},{ }_{-}=254 \mathrm{~nm}, \mathrm{t}_{\mathrm{R}}=18.5 \mathrm{~min}$, minor; $\mathrm{t}_{\mathrm{R}}=19.5 \mathrm{~min}$, major).

\section{Compound (15)}

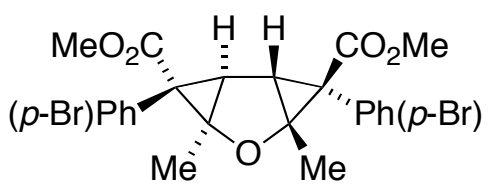

15

A solution of methyl para-bromophenyldiazoacetate 4 (191 mg, $0.75 \mathrm{mmol}, 2.5 \mathrm{eq}$.) in hexanes $(10 \mathrm{~mL})$ was added by syringe pump over $1 \mathrm{~h}$ to a solution of 2,5-dimethylfuran 14 (28 mg, $0.30 \mathrm{mmol}, 1$ eq.) and $\mathrm{Rh}_{2}(S-\mathrm{DOSP})_{4}(4 \mathrm{mg}, 0.003 \mathrm{mmol}, 0.01$ eq. $)$ in hexane $(5 \mathrm{~mL})$. The reaction mixture was stirred for an additional $1 \mathrm{~h}$, and then concentrated in vacuo. Purified by flash chromatography on silica gel using 9:1 to $5: 1$ hexane/Et ${ }_{2} \mathrm{O}$ as eluant to isolate a white solid $125 \mathrm{mg}(76 \%) . \mathrm{mp} 208-211{ }^{\circ} \mathrm{C}$. [ $]_{\mathrm{D}} 76.2(84 \%$ ee) $(c 1.00$ $\left.\mathrm{CHCl}_{3}\right) .{ }^{1} \mathrm{H} \mathrm{NMR}\left(300 \mathrm{MHz}, \mathrm{CDCl}_{3}\right):{ }_{-} .54(\mathrm{~d}, 4 \mathrm{H}, J=7.5 \mathrm{~Hz}), 7.17$ (d, 4H, J=7.5 Hz), 
$3.53(\mathrm{~s}, 6 \mathrm{H}), 2.67$ (s, 2H), 0.49 (s, 6H). ${ }^{13} \mathrm{C}$ NMR $\left(75 \mathrm{MHz}, \mathrm{CDCl}_{3}\right)$ :_170.5, 131.9, 131.4 121.7, 73.4, 52.6, 41.4, 37.3, 13.0. IR $\left(\mathrm{CHCl}_{3}\right): 2924,1732,1717,1486,1430,1394$, 1301, 1233, 1196, 1171, 1080, 1069, $1011 \mathrm{~cm}^{-1} \cdot m / z(E I): 552\left(\mathrm{M}^{81} \mathrm{Br}_{2}{ }^{+}, 17 \%\right), 550$ $\left(\mathrm{M}^{81} \mathrm{Br}^{79} \mathrm{Br}^{+}, 30 \%\right), 548\left(\mathrm{M}^{79} \mathrm{Br}_{2}^{+}, 16 \%\right), 520$ (32\%), 518 (62\%), $516(31 \%), 443$ (100\%), 415 (53\%), 228 (68\%). Anal. Calcd. for $\mathrm{C}_{24} \mathrm{H}_{22} \mathrm{Br}_{2} \mathrm{O}_{5}$ : C 52.39; $\mathrm{H}$ 4.03, found $\mathrm{C} 52.38 ; \mathrm{H}$ 3.99. HPLC analysis: $84 \%$ ee (Chiralcel OD-H, $1.0 \% i-\mathrm{PrOH}$ in hexane, $0.8 \mathrm{ml} / \mathrm{min}$, $254 \mathrm{~nm}, \mathrm{t}_{\mathrm{R}}=10.2 \mathrm{~min}$, major; $\mathrm{t}_{\mathrm{R}}=13.4 \mathrm{~min}$, minor).

(2E,4Z)-Dimethyl 2-(4-bromophenyl)hexa-2,4-dienedioate (17a) and (2Z,4Z)Dimethyl 2-(4-bromophenyl)hexa-2,4-dienedioate (17b)

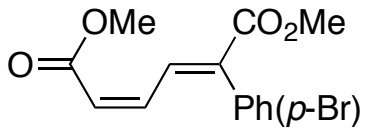

$17 a$

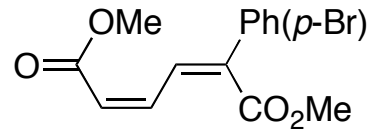

$17 b$

A solution of methyl para-bromophenyldiazoacetate 4 (255 mg, $1.0 \mathrm{mmol}, 1.0 \mathrm{eq}$.) in hexanes $(10 \mathrm{~mL})$ was added by syringe pump over $1 \mathrm{~h}$ to a solution of 2-methoxyfuran $\mathbf{1 6}$ (0.55 mL, $6.0 \mathrm{mmol}, 6.0$ eq. $)$ and $\mathrm{Rh}_{2}(S \text {-DOSP })_{4}(10.0 \mathrm{mg}, 0.01 \mathrm{mmol}, 0.01$ eq. $)$ in hexane $(5 \mathrm{~mL})$. The reaction mixture was stirred for an additional $1 \mathrm{~h}$, and then concentrated in vacuo. Purified by flash chromatography on silica gel using 9:1 hexane/Et $t_{2} \mathrm{O}$ as eluant to isolate yellow solid 17a, $280 \mathrm{mg}(86 \%)$; and yellow oil 17b, 29 mg $(9 \%)$.

Compound 17a: Mp 91-94 ${ }^{\circ} \mathrm{C} .{ }^{1} \mathrm{H}$ NMR (400 MHz, $\left.\mathrm{CDCl}_{3}\right)$ :_8.57 (d, $\left.1 \mathrm{H}, J=11.5 \mathrm{~Hz}\right)$, $7.52(\mathrm{~d}, 2 \mathrm{H}, J=8.5 \mathrm{~Hz}), 7.09$ (d, 2H, $J=8.5 \mathrm{~Hz}), 6.55(\mathrm{t}, 1 \mathrm{H}, J=11.5 \mathrm{~Hz}), 5.90(\mathrm{~d}, 1 \mathrm{H}$, $J=11.5 \mathrm{~Hz}), 3.82(\mathrm{~s}, 3 \mathrm{H}), 3.79(\mathrm{~s}, 3 \mathrm{H}) .{ }^{13} \mathrm{C}$ NMR $\left(75 \mathrm{MHz}, \mathrm{CDCl}_{3}\right): \_166.7,165.8$, 138.4, 138.1, 134.1, 132.7, 131.8, 131.2, 124.1, 122.7, 52.4, 51.5. IR $\left(\mathrm{CHCl}_{3}\right): 3093$, $2950,1719,1701,1623,1578,1487,1436,1262,1238,1194,1172,1098,1069,1037$, 1008, 991, 943, 922, 902, 826, 795, 777, 767, 723, $623 \mathrm{~cm}^{-1} . \mathrm{m} / z$ (ESI): $349\left(\mathrm{M}^{81} \mathrm{BrNa}^{+}\right.$, 
61\%), $347\left(\mathrm{M}^{79} \mathrm{MNa}^{+}, 46 \%\right), 344$ (100\%), 342 (96\%), 327 (48\%), 325 (44\%). HRMS Calcd. for $\mathrm{C}_{14} \mathrm{H}_{13} \mathrm{O}_{4} \mathrm{BrNa}\left(\mathrm{MNa}^{+}\right)$: 346.9889 . Found: 346.9882 .

Compound 17b: ${ }^{1} \mathrm{H}$ NMR (400 MHz, $\left.\mathrm{CDCl}_{3}\right)$ : $8.14(\mathrm{~d}, 1 \mathrm{H}, J=12.5 \mathrm{~Hz}), 7.53$ (d, 2H, $J=8.5 \mathrm{~Hz}), 7.35(\mathrm{~d}, 2 \mathrm{H}, J=8.5 \mathrm{~Hz}), 7.17(\mathrm{t}, 1 \mathrm{H}, J=11.0 \mathrm{~Hz}), 5.98(\mathrm{~d}, 1 \mathrm{H}, J=11.0 \mathrm{~Hz}), 3.92$ (s, 3H), $3.81(\mathrm{~s}, 3 \mathrm{H}) .{ }^{13} \mathrm{C} \mathrm{NMR}\left(75 \mathrm{MHz}, \mathrm{CDCl}_{3}\right)$ :_167.5, 166.3, 139.4, 139.0, 135.3, 131.7, 129.2, 129.0, 123.3, 122.0, 52.3, 51.5. IR $\left(\mathrm{CHCl}_{3}\right): 2952,1716,1620,1583,1488$, $1435,1338,1197,1174,1073,1009,824,769,751 \mathrm{~cm}^{-1} . m / z(E I): 326\left(\mathrm{M}^{81} \mathrm{Br}^{+}, 23 \%\right)$, $324\left(\mathrm{M}^{79} \mathrm{Br}^{+}, 23 \%\right), 267$ (99\%), 265 (100\%), 235 (52\%), 233 (52\%). Anal. Calcd. for $\mathrm{C}_{14} \mathrm{H}_{13} \mathrm{BrO}_{4}$ : C, 51.71; H, 4.03. Found: C, 51.85; H, 3.92.

(1R,1aS,6aS)-1-(4-Bromo-phenyl)-1a,6a-dihydro-1H-6-oxa-cyclopropa[a]indene-1carboxylic acid methyl ester (20)

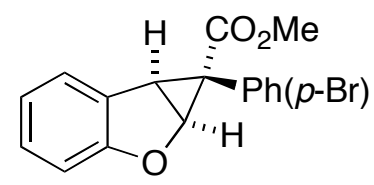

20

A solution of methyl para-bromophenyldiazoacetate 4 (128 mg, $0.5 \mathrm{mmol}, 1.00 \mathrm{eq}$.) in hexanes $(5 \mathrm{~mL})$ was added by syringe pump over $1 \mathrm{~h}$ to a solution of 2,3-benzofuran $\mathbf{1 8}$ (400 mg, $3.39 \mathrm{mmol}, 6.77$ eq.) and $\mathrm{Rh}_{2}(S \text {-DOSP })_{4}(9.5 \mathrm{mg}, 0.005 \mathrm{mmol}, 0.01$ eq.) in hexane $(5 \mathrm{~mL})$. The reaction mixture was stirred for an additional $1 \mathrm{~h}$, and then concentrated in vacuo. Purified by flash chromatography on silica gel using 20:1 hexane/Et ${ }_{2} \mathrm{O}$, then $10: 1$ hexane/ $\mathrm{Et}_{2} \mathrm{O}$ to provide the title compound as a white solid, 105 $\operatorname{mg}(61 \%)$. Mp 128-130 ${ }^{\circ} \mathrm{C} .[]_{\mathrm{D}}-90.2\left(96 \%\right.$ ee) $\left(c\right.$ 1.49, $\left.\mathrm{CHCl}_{3}\right) .{ }^{1} \mathrm{H}$ NMR $(500 \mathrm{MHz}$, $\left.\mathrm{CDCl}_{3}\right)$ : $7.34(\mathrm{~d}, 1 \mathrm{H}, J=7.0 \mathrm{~Hz}), 7.21(\mathrm{~d}, 2 \mathrm{H}, J=8.5 \mathrm{~Hz}), 6.99-6.92(\mathrm{~m}, 3 \mathrm{H}), 6.83(\mathrm{t}, 1 \mathrm{H}$, $J=7.5 \mathrm{~Hz}), 6.50(\mathrm{~d}, 1 \mathrm{H}, J=8.0 \mathrm{~Hz}), 5.35(\mathrm{~d}, 1 \mathrm{H}, J=5.5 \mathrm{~Hz}), 3.79(\mathrm{~d}, 1 \mathrm{H}, J=5.5 \mathrm{~Hz}), 3.67$ (s, 3H). ${ }^{13} \mathrm{C}$ NMR $\left(75 \mathrm{MHz}, \mathrm{CDCl}_{3}\right)$ :_172.7, 159.4, 134.1, 130.8, 128.8, 128.3, 126.1, 
124.9, 121.5, 121.4, 109.9, 70.3, 52.8, 37.4, 30.4. IR $\left(\mathrm{CHCl}_{3}\right): 3055,2951,1711,1593$, 1466, 1258, 1243, 1035, $751 \mathrm{~cm}^{-1} . \mathrm{m} / z$ (ESI): $369\left(\mathrm{M}^{81} \mathrm{BrNa}^{+}, 83 \%\right), 367\left(\mathrm{M}^{79} \mathrm{BrMNa}^{+}\right.$, 83\%), 315 (33\%)287 (31\%), 313 (30\%), 286 (34\%), 234 (44\%). Anal. Calcd. for $\mathrm{C}_{17} \mathrm{H}_{13} \mathrm{BrO}_{3}$ : C, 59.15; H, 3.80. Found: $\mathrm{C}, 59.30 ; \mathrm{H}, 3.80$. HPLC analysis: $96 \%$ ee (Chiralcel OD-H, $1 \%{ }^{i} \mathrm{PrOH}, 1 \mathrm{ml} / \mathrm{min},{ }_{-}=254 \mathrm{~nm}, t_{\mathrm{R}}=9.9 \mathrm{~min}$ (minor); $t_{\mathrm{R}}=11.4$ (major)).

(1S,1aS,6aS)-1-(4-Bromo-phenyl)-1a-methyl-1a,6a-dihydro-1H-6-oxacyclopropa[a]indene-1-carboxylic acid methyl ester (21)

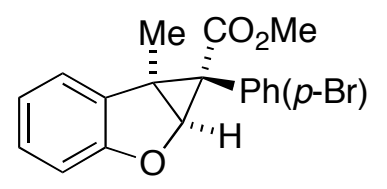

21

A solution of methyl para-bromophenyldiazoacetate 4 (236 mg, $0.93 \mathrm{mmol}, 1.00$ eq.) in hexanes $(5 \mathrm{~mL})$ was added by syringe pump over $1 \mathrm{~h}$ to a solution of 3-methylbenzofuran 19 (367 mg, $2.77 \mathrm{mmol}, 3.00$ eq.) and $\mathrm{Rh}_{2}(S \text {-DOSP })_{4}(18 \mathrm{mg}, 0.009 \mathrm{mmol}, 0.01$ eq.) in hexane $(5 \mathrm{~mL})$. The reaction mixture was stirred for an additional $1 \mathrm{~h}$, and then concentrated in vacuo. Purified by flash chromatography on silica gel using 20:1 hexane/ $/ \mathrm{t}_{2} \mathrm{O}$, then 10:1 hexane/ $\mathrm{Et}_{2} \mathrm{O}$ to provide the title compound as a colorless gum, $271 \mathrm{mg}(81 \%)$. $[\alpha]_{\mathrm{D}}-22.8\left(97 \%\right.$ ee) (c 1.14, $\left.\mathrm{CHCl}_{3}\right) .{ }^{1} \mathrm{H}$ NMR $\left(400 \mathrm{MHz}, \mathrm{CDCl}_{3}\right)$ : _ 7.29-7.25 (m, 1H), 7.18 (d, 2H, J=8.5 Hz), 6.98-6.89 (m, 3H), 6.86 (t, 1H, J=7.0 Hz), $6.48(\mathrm{~d}, 1 \mathrm{H}, J=8.0 \mathrm{~Hz}), 5.44$ (s, 1H), 3.69 (s, 3H), 1.84 (s, 3H). ${ }^{13} \mathrm{C}$ NMR $(75 \mathrm{MHz}$, $\left.\mathrm{CDCl}_{3}\right): \delta 171.2,158.5,133.8,130.5,130.3(2 \mathrm{C}), 128.1,123.6(2 \mathrm{C}), 121.1,109.7,73.3$, 52.4, 42.2, 34.8, 12.3. IR $\left(\mathrm{CHCl}_{3}\right): 2951,2928,2848,1713,1475,1250,1176,1041,750$ $\mathrm{cm}^{-1} . \mathrm{m} / \mathrm{z}$ (EI): $360\left(\mathrm{M}^{81} \mathrm{Br}^{+}, 32 \%\right), 358\left(\mathrm{M}^{79} \mathrm{Br}^{+}, 34 \%\right), 301$ (92\%), 299 (100\%), 219 (51\%). Anal. Calcd. for $\mathrm{C}_{18} \mathrm{H}_{15} \mathrm{BrO}_{3}$ : C, 60.18; H, 4.21. Found: C, 60.01; H, 4.18. HPLC 
analysis: $97 \%$ ee (Chiralcel OD-H, $1 \%{ }^{i} \mathrm{PrOH}, 1 \mathrm{ml} / \mathrm{min},{ }_{-}=254 \mathrm{~nm}, t_{\mathrm{R}}=7.2 \mathrm{~min}$ (minor); $t_{\mathrm{R}}=9.2$ (major)).

\section{Compound (23)}

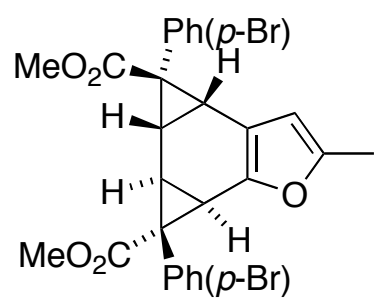

23

Procedure A: A solution of methyl para-bromophenyldiazoacetate 4 (128 mg, $0.5 \mathrm{mmol}$, 1.00 eq.) in hexanes $(5 \mathrm{~mL})$ was added by syringe pump over $1 \mathrm{~h}$ to a solution of 2 methylbenzofuran 22 (198 mg, $1.5 \mathrm{mmol}, 3.0$ eq.) and $\mathrm{Rh}_{2}(S \text {-DOSP })_{4}(9.5 \mathrm{mg}, 0.005$ mmol, 0.01 eq.) in hexane $(5 \mathrm{~mL})$. The reaction mixture was stirred for an additional $1 \mathrm{~h}$, and then concentrated in vacuo. Purified by flash chromatography on silica gel using 20:1 hexane/Et ${ }_{2} \mathrm{O}$, then $10: 1$ hexane/Et ${ }_{2} \mathrm{O}$ to provide the title compound as a white solid, 138 $\operatorname{mg}(47 \%)$.

Procedure B: A solution of methyl para-bromophenyldiazoacetate 4 (128 mg, $0.5 \mathrm{mmol}$, 1.00 eq.) in hexanes $(5 \mathrm{~mL})$ was added by syringe pump over $1 \mathrm{~h}$ to a solution of 2methylbenzofuran $22\left(198 \mathrm{mg}, 1.5 \mathrm{mmol}, 3.0\right.$ eq.) and $\mathrm{Rh}_{2}(S \text {-DOSP })_{4}(9.5 \mathrm{mg}, 0.005$ mmol, 0.01 eq.) in hexane $(5 \mathrm{~mL})$ under reflux. The reaction mixture was stirred at reflux for an additional $1 \mathrm{~h}$, ten allowed to cool to room temperature and concentrated in vacuo. Purified by flash chromatography on silica gel using $20: 1$ hexane/Et $t_{2} \mathrm{O}$, then $10: 1$ hexane/ $\mathrm{Et}_{2} \mathrm{O}$ to provide the title compound as a white solid, $144 \mathrm{mg}(49 \%)$.

Compound 23: $\mathrm{Mp} 201-202{ }^{\circ} \mathrm{C} .[\alpha]_{\mathrm{D}} 19.0\left(51 \%\right.$ ee) $\left(c\right.$ 1.05, $\left.\mathrm{CHCl}_{3}\right) .{ }^{1} \mathrm{H} \mathrm{NMR}(400 \mathrm{MHz}$, $\left.\mathrm{CDCl}_{3}\right): \delta 7.36(\mathrm{~d}, 2 \mathrm{H}, J=9.0 \mathrm{~Hz}), 7.33(\mathrm{~d}, 2 \mathrm{H}, J=9.0 \mathrm{~Hz}), 6.92(\mathrm{~d}, 2 \mathrm{H}, J=9.0 \mathrm{~Hz}), 6.83(\mathrm{~d}$, 
$2 \mathrm{H}, J=9.0 \mathrm{~Hz}), 5.70$ (s, 1H), 3.60 (s, 3H), 3.59 (s, 3H), 2.71 (d, 1H, $J=9.0 \mathrm{~Hz}), 2.57$ (d, $1 \mathrm{H}, J=9.0 \mathrm{~Hz}), 2.23(\mathrm{~s}, 3 \mathrm{H}), 2.15(\mathrm{~d}, 1 \mathrm{H}, J=9.0 \mathrm{~Hz}), 1.98(\mathrm{~d}, 1 \mathrm{H}, J=9.0 \mathrm{~Hz}) \cdot{ }^{13} \mathrm{C}$ NMR (75 MHz, $\left.\mathrm{CDCl}_{3}\right): \delta 172.8,172.2,151.9,144.1,134.0,133.4,132.4,132.3,131.1$ (2C), $121.4,121.3,114.8,106.2,52.7,52.6,39.3,38.6,28.1,26.9,25.2,25.1,13.7$. IR $\left(\mathrm{CHCl}_{3}\right): 2951,2920,2848,1713,1433,1234,1011,735 \mathrm{~cm}^{-1} . \mathrm{m} / \mathrm{z}$ (ESI): $587\left(\mathrm{MH}^{+}\right.$, 100\%), 566 (22\%), 493 (24\%), 121 (32\%), 105 (36\%). HRMS Calcd. for $\mathrm{C}_{27} \mathrm{H}_{23} \mathrm{O}_{5}{ }^{79} \mathrm{Br}^{81} \mathrm{Br}\left(\mathrm{MH}^{+}\right)$: 586.9886. Found: 586.9890. HPLC analysis: $51 \%$ ee (Chiralcel OD-H, $2 \%{ }^{i} \mathrm{PrOH}, 1 \mathrm{ml} / \mathrm{min},{ }_{-}=254 \mathrm{~nm}, t_{\mathrm{R}}=13.1 \mathrm{~min}$ (minor); $t_{\mathrm{R}}=15.8$ (major)).

\section{Compound (26)}

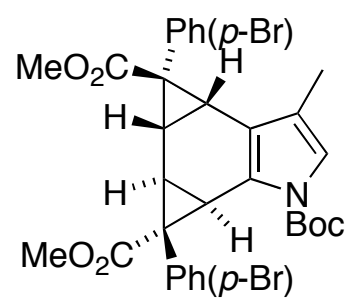

26

A solution of methyl para-bromophenyldiazoacetate 4 (374 mg, $1.46 \mathrm{mmol}, 2.2 \mathrm{eq}$.) in hexanes $(10 \mathrm{~mL})$ was added by syringe pump over $5 \mathrm{~h}$ to a solution of tert-butyl 3-methyl$1 H$-indole-1-carboxylate $\mathbf{2 4}^{4}(154 \mathrm{mg}, 0.67 \mathrm{mmol}, 1.0$ eq. $)$ and $\mathrm{Rh}_{2}(S \text {-DOSP })_{4}(9.5 \mathrm{mg}$, $0.005 \mathrm{mmol}, 0.01$ eq.) in hexane $(5 \mathrm{~mL})$. The reaction mixture was stirred for an additional $1 \mathrm{~h}$, and then concentrated in vacuo. Purified by flash chromatography on silica gel using 20:1 hexane/Et $\mathrm{t}_{2} \mathrm{O}$, then 10:1 hexane/Et ${ }_{2} \mathrm{O}$ to provide the title compound as a white solid, $390 \mathrm{mg}(85 \%)$. Mp $168-170{ }^{\circ} \mathrm{C}$ (decomposes). $[\alpha]_{\mathrm{D}}-12.0$ (79\% ee) (c 1.22, $\left.\mathrm{CHCl}_{3}\right) .{ }^{1} \mathrm{H}$ NMR $\left(400 \mathrm{MHz}, \mathrm{CDCl}_{3}\right):$ _ 7.38-7.30 (m, 4H), 6.88-6.80 (m, 5H), $3.61(\mathrm{~s}$, $3 \mathrm{H}), 3.58$ (s, 3H), 2.66 (d, 1H, $J=9.5 \mathrm{~Hz}), 2.62$ (d, $1 \mathrm{H}, J=9.5 \mathrm{~Hz}), 2.53$ (d, 1H, $J=9.5 \mathrm{~Hz}$ ), 2.04 (d, $1 \mathrm{H}, J=9.5 \mathrm{~Hz}), 1.89$ (s, 3H), 1.52 (s, 9H). ${ }^{13} \mathrm{C} \mathrm{NMR}\left(75 \mathrm{MHz}, \mathrm{CDCl}_{3}\right)$ :_173.5, 
173.0, 149.4, 134.4, 133.7, 133.4, 132.9, 131.7, 131.6, 124.2, 121.8, 121.7, 120.4, 119.1, $118.7,84.5,53.2,53.1,39.5,39.3,30.2,28.5,28.0,27.4,27.0,24.9$. IR $\left(\mathrm{CHCl}_{3}\right): 2980$, 2952, 2928, 2858, 1714, 1587, 1370, 1360, 1235, 1155, 1071, $730 \mathrm{~cm}^{-1} . \mathrm{m} / z$ (EI): 685 $\left(\mathrm{M}^{+}, 5 \%\right), 597$ (44\%), 537 (59\%), 185 (33\%), 57 (100\%). HRMS (EI) Calcd. for $\mathrm{C}_{32} \mathrm{H}_{31} \mathrm{O}_{6} \mathrm{~N}^{79} \mathrm{Br}^{81} \mathrm{Br}$ : 685.0492. Found: 685.0518. HPLC analysis: 79\% ee (Chiralcel OD$\mathrm{H}, 0.4 \%{ }^{i} \mathrm{PrOH}, 0.7 \mathrm{ml} / \mathrm{min},{ }_{-}=254 \mathrm{~nm}, t_{\mathrm{R}}=48.6 \mathrm{~min}$ (major); $t_{\mathrm{R}}=63.1$ (minor)).

\section{Compound (27)}

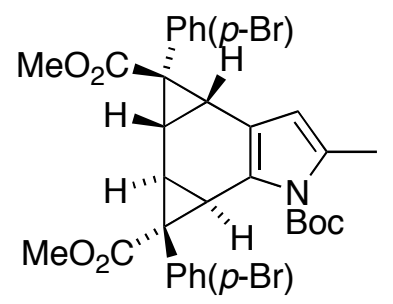

27

A solution of methyl para-bromophenyldiazoacetate 4 (742 mg, $2.91 \mathrm{mmol}, 2.2 \mathrm{eq}$.) in hexanes $(15 \mathrm{~mL})$ was added by syringe pump over $5 \mathrm{~h}$ to a solution of tert-butyl 2-methyl$1 H$-indole-1-carboxylate $\mathbf{2 5}^{4}$ (306 mg, $1.32 \mathrm{mmol}, 1.0$ eq.) and $\mathrm{Rh}_{2}(S \text {-DOSP) })_{4}(55 \mathrm{mg}$, $0.03 \mathrm{mmol}, 0.02$ eq.) in hexane $(5 \mathrm{~mL})$. The reaction mixture was stirred for an additional 1h, and then concentrated in vacuo. Purified by flash chromatography on silica gel using 20:1 hexane/ $\mathrm{Et}_{2} \mathrm{O}$, then 10:1 hexane/ $\mathrm{Et}_{2} \mathrm{O}$ to provide the title compound as a white solid, $513 \mathrm{mg}(61 \%) . \mathrm{mp} 185-191{ }^{\circ} \mathrm{C}$ (decomposes). $[\alpha]_{\mathrm{D}} 18.4\left(87 \%\right.$ ee) $\left(c \quad 0.98, \mathrm{CHCl}_{3}\right) .{ }^{1} \mathrm{H}$ NMR ( $\left.\mathrm{CDCl}_{3}, 400 \mathrm{MHz}\right): \delta 7.38-7.31(\mathrm{~m}, 4 \mathrm{H}), 6.90$ (d, 2H, $\left.J=8.0 \mathrm{~Hz}\right), 6.86$ (d, 2H, $J=8.0$ Hz), $5.61(\mathrm{~s}, 1 \mathrm{H}), 3.59(\mathrm{~s}, 3 \mathrm{H}), 3.57(\mathrm{~s}, 3 \mathrm{H}), 2.61(\mathrm{~d}, 1 \mathrm{H}, J=10.0 \mathrm{~Hz}), 2.57$ (d, 1H, J=9.5 $\mathrm{Hz}), 2.46(\mathrm{~d}, 1 \mathrm{H}, J=10.0 \mathrm{~Hz}), 2.36(\mathrm{~s}, 3 \mathrm{H}), 2.03(\mathrm{~d}, 1 \mathrm{H}, J=9.5 \mathrm{~Hz}), 1.51(\mathrm{~s}, 9 \mathrm{H}) .{ }^{13} \mathrm{C}$ NMR ( $\left.\mathrm{CDCl}_{3}, 75 \mathrm{MHz}\right): 173.5,173.2,150.2,134.5,133.8,133.5,133.4,133.0,131.6$, $131.5,124.0,121.7,121.6,116.8,111.9,84.7,53.2,53.0,39.3,39.1,28.5,28.3,28.2$, 
27.0, 26.5, 16.7. IR $\left(\mathrm{CHCl}_{3}\right): 2890,2951,2922,2848,1713,1593,1548,1348,1234$, 1120, 908, $729 \mathrm{~cm}^{-1} . m / z$ (ESI): $710\left(\mathrm{M}^{81} \mathrm{Br}_{2} \mathrm{Na}^{+}, 53 \%\right), 708\left(\mathrm{M}^{81} \mathrm{Br}^{79} \mathrm{BrNa}^{+}, 100 \%\right), 706$ $\left(\mathrm{M}^{79} \mathrm{Br}_{2} \mathrm{Na}^{+}\right), 585$ (28\%). HRMS Calcd. For $\mathrm{C}_{12} \mathrm{H}_{31} \mathrm{O}_{6} \mathrm{NBr}_{2} \mathrm{Na}\left(\mathrm{MNa}^{+}\right): 706.0410$. Found: 706.0407. HPLC analysis: $87 \%$ ee (Chiralcel OD-H, $0.4 \%{ }^{i} \mathrm{PrOH}, 0.6 \mathrm{ml} / \mathrm{min},{ }_{-}=254$ $\mathrm{nm}, t_{\mathrm{R}}=40.0 \min \left(\right.$ minor); $t_{\mathrm{R}}=45.1$ (major)).

\section{tert-Butyl 3-((tert-butyldimethylsilyloxy)methyl)-1H-indole-1-carboxylate (28)}

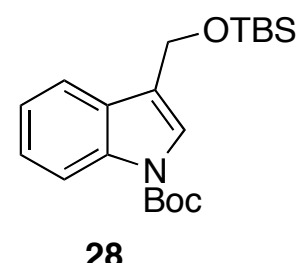

A solution of tert-butyl 3-(hydroxymethyl)-1H-indole-1-carboxylate ${ }^{5}(1.88 \mathrm{~g}, 7.25 \mathrm{mmol}$, 1.0 eq.) and tert-butyldimethylsilyl chloride (1.20 g, $7.98 \mathrm{mmol}, 1.1$ eq.) in dichloromethane $(50 \mathrm{~mL})$ was stirred until homogeneous, and imidazole $(592 \mathrm{mg}, 8.70$ mmol, 1.2 eq.) was added. A white precipitate formed immediately, and was stirred for 6 h. The reaction mixture was filtered through a plug of silica, and then concentrated in vacuo. The residue was purified by flash chromatography using $20: 1$ hexane/Et $2 \mathrm{O}$ to provide a colorless oil, 2.40g (92\%). ${ }^{1} \mathrm{H}$ NMR $\left(500 \mathrm{MHz}, \mathrm{CDCl}_{3}\right)$ :_8.13 (br s, $\left.1 \mathrm{H}\right), 7.58$ (d, 1H, $J=7.5 \mathrm{~Hz}), 7.52$ (s, 1H), 7.32 (t, 1H, $J=7.5 \mathrm{~Hz}), 7.24$ (t, 1H, $J=7.5 \mathrm{~Hz}), 4.88$ (s, 2H), 1.67 (s, 9H), 0.95 (s, 9H), 0.13 (s, 6H). ${ }^{13} \mathrm{C} \mathrm{NMR}\left(75 \mathrm{MHz}, \mathrm{CDCl}_{3}\right): \delta 149.8,135.8$, 129.3, 124.3, 122.9, 122.4, 121.1, 119.5, 115.2, 83.4, 58.0, 28.2, 25.9, 18.4, -5.3. IR $\left(\mathrm{CHCl}_{3}\right): 2955,2929,2852,1734,1452,1354,1255,1159,1088,1066,837,769,747$ $\mathrm{cm}^{-1} \cdot \mathrm{m} / z$ (ESI): $384\left(\mathrm{MNa}^{+}, 33 \%\right), 347$ (32\%), 230 (100\%). Anal. Calcd. for $\mathrm{C}_{20} \mathrm{H}_{31} \mathrm{O}_{3} \mathrm{NSi}$ : C, 66.44; H, 8.64; N, 3.87. Found: C, 66.58; H, 8.66; N, 3.89. 
tert-Butyl 3-((1S,2S)-1-(tert-butyldimethylsilyloxy))-3-methoxy-3-oxo-2phenylpropyl)-1 $H$-indole-1-carboxylate (30) and Compound (31)

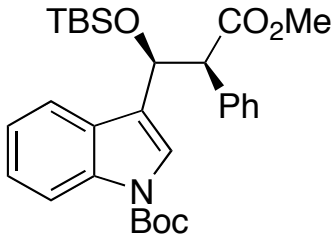

30

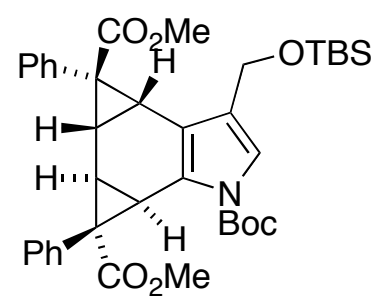

31

Procedure A: A solution of methyl phenyldiazoacetate 29 (176 mg, $1.00 \mathrm{mmol}, 2.00$ eq.) in 2,2-dimethylbutane $(9 \mathrm{~mL})$ was added via syringe pump over $2 \mathrm{~h}$ to a solution of compound 28 (187 mg, $0.5 \mathrm{mmol}, 1.00$ eq.), $\mathrm{Rh}_{2}(S \text {-DOSP })_{4}(8.5 \mathrm{mg}, 0.005 \mathrm{mmol}, 0.005$ eq.) and 2,2-dimethylbutane $(5 \mathrm{~mL})$. The reaction mixture was stirred for $1 \mathrm{~h}$, and then concentrated in vacuo. Crude NMR showed 91\%de syn/anti. Purified by flash chromatography on silica gel using 20:1 hexane/ $\mathrm{Et}_{2} \mathrm{O}$ as eluant to isolate the anti isomer as a colorless oil, $5 \mathrm{mg}$, a mixture of the anti and $s y n$ isomers as a colorless oil, $21 \mathrm{mg}$, and the syn isomer as a colorless oil, $38 \mathrm{mg}$. Total mass: $64 \mathrm{mg}(25 \%)$. Also isolated compound 31 as a white solid, $216 \mathrm{mg}(66 \%)$.

Procedure B: A solution of methyl phenyldiazoacetate 29 (176 mg, $1.00 \mathrm{mmol}, 2.00$ eq.) in 2,2-dimethylbutane $(9 \mathrm{~mL})$ was added via syringe pump over $2 \mathrm{~h}$ to a solution of compound 28 (187 mg, $0.5 \mathrm{mmol}, 1.00$ eq.), $\mathrm{Rh}_{2}(\mathrm{OOct})_{4}(7.8 \mathrm{mg}, 0.01 \mathrm{mmol}, 0.02$ eq.) and 2,2-dimethylbutane $(5 \mathrm{~mL})$. The reaction mixture was stirred for $1 \mathrm{~h}$, and then concentrated in vacuo. Crude NMR showed $87 \%$ de syn/anti. Purified by flash chromatography on silica gel using 20:1 hexane/ $\mathrm{Et}_{2} \mathrm{O}$ as eluant to isolate the anti isomer as a colorless oil, $3 \mathrm{mg}$, a mixture of the anti and syn isomers as a colorless oil, $86 \mathrm{mg}$, and the syn isomer as a colorless oil, $93 \mathrm{mg}$. Total mass: $182 \mathrm{mg}(70 \%)$. 
Procedure C: A solution of methyl phenyldiazoacetate 29 (193 mg, $1.10 \mathrm{mmol}, 2.20$ eq.) in 2,2-dimethylbutane $(6 \mathrm{~mL})$ was added via syringe pump over $3 \mathrm{~h}$ to a solution of compound 28 (187 mg, 0.5 mmol, 1.00 eq.), $\mathrm{Rh}_{2}(S-\mathrm{PTTL})_{4}(13.6 \mathrm{mg}, 0.011 \mathrm{mmol}, 0.02$ eq.) and 2,2-dimethylbutane $(5 \mathrm{~mL})$ heated under reflux. The reaction mixture was stirred at reflux for $30 \mathrm{~min}$, then allowed to cool to room temperature and concentrated in vacuo. Purified by flash chromatography on silica gel using 5:1 hexane/Et $t_{2} \mathrm{O}$ as eluant to isolate product ent-31 as a white solid, $302 \mathrm{mg}(92 \%)$. No C-H activation product 30 was isolated in this reaction.

Compound 30: anti isomer: ${ }^{1} \mathrm{H}$ NMR (500 MHz, $\mathrm{CDCl}_{3}$ ):_8.02 (br s, $\left.1 \mathrm{H}\right), 7.70$ (br s, 1H), 7.34-7.24 (m, 3H), 7.19-7.01 (m, 5H), 5.37 (d, 1H, $J=10.5 \mathrm{~Hz}), 4.21$ (d, 1H, $J=10.5$ $\mathrm{Hz}), 3.75(\mathrm{~s}, 3 \mathrm{H}), 1.61(\mathrm{~s}, 9 \mathrm{H}), 0.85(\mathrm{~s}, 9 \mathrm{H}), 0.11(\mathrm{~s}, 3 \mathrm{H}),-0.28(\mathrm{~s}, 3 \mathrm{H})$. syn isomer : $[\alpha]_{\mathrm{D}}$ $17.3\left(36 \%\right.$ ee) $\left(c\right.$ 1.11, $\left.\mathrm{CHCl}_{3}\right) .{ }^{1} \mathrm{H}$ NMR $\left(500 \mathrm{MHz}, \mathrm{CDCl}_{3}\right): \_8.03(\mathrm{br} \mathrm{s}, 1 \mathrm{H}), 7.82(\mathrm{~d}$, 1H, $J=9.0 \mathrm{~Hz}), 7.51-7.44(\mathrm{~m}, 3 \mathrm{H}), 7.39-7.22(\mathrm{~m}, 5 \mathrm{H}), 5.43$ (d, 1H, J=9.0 Hz), 4.13 (d, 1H, $J=9.0 \mathrm{~Hz}$ ), 3.46 (s, 3H), 1.68 (s, 9H), 0.61 (s, 9H), -0.32 (s, 3H), -.0.37 (s, 3H). ${ }^{13} \mathrm{C}$ NMR (75 MHz, $\left.\mathrm{CDCl}_{3}\right): \delta 172.1,149.7,136.4,135.7,129.4,128.5,128.2,127.4,124.2$, 123.6, 122.4, 122.2, 120.8, 115.1, 83.6, 71.0, 59.9, 51.7, 28.2, 25.4, 17.8, -5.1, -5.9. IR $\left(\mathrm{CHCl}_{3}\right): 2953,2929,2887,2856,1734,1450,1369,1254,1157,1088,837,777,698$ $\mathrm{cm}^{-1} . \mathrm{m} / z$ (ESI): $532\left(\mathrm{M}^{+}, 100 \%\right), 476(45 \%), 378$ (19\%), 322 (29\%). Anal. Calcd. for $\mathrm{C}_{29} \mathrm{H}_{39} \mathrm{NO}_{5} \mathrm{Si}: \mathrm{C}, 68.34 ; \mathrm{H}, 7.71 ; \mathrm{N}, 2.75$. Found: $\mathrm{C}, 68.62 ; \mathrm{H}, 7.91 ; \mathrm{N}, 2.62$. HPLC Analysis: $31 \%$ ee (Chiralcel OD-H, $1 \%{ }^{i} \mathrm{PrOH}, 1 \mathrm{ml} / \mathrm{min},{ }_{-}=254 \mathrm{~nm}, t_{\mathrm{R}}=4.4 \mathrm{~min}$ (major); $t_{\mathrm{R}}=5.0$ (minor)).

Compound ent-31: $\mathrm{Mp} 70-72{ }^{\circ} \mathrm{C} .[\alpha]_{\mathrm{D}}-18.7\left(94 \%\right.$ ee) $\left(c\right.$ 1.89, $\left.\mathrm{CHCl}_{3}\right) .{ }^{1} \mathrm{H}$ NMR $(500$ $\left.\mathrm{MHz}, \mathrm{CDCl}_{3}\right)$ : $7.23-7.13(\mathrm{~m}, 8 \mathrm{H}), 6.99(\mathrm{~s}, 1 \mathrm{H}), 6.98-6.94(\mathrm{~m}, 2 \mathrm{H}), 4.36(\mathrm{~s}, 2 \mathrm{H}), 3.57(\mathrm{~s}$, 
$3 \mathrm{H}), 3.56(\mathrm{~s}, 3 \mathrm{H}), 2.71(\mathrm{~d}, 1 \mathrm{H}, J=9.5 \mathrm{~Hz}), 2.68(\mathrm{~d}, 1 \mathrm{H}, J=9.5 \mathrm{~Hz}), 2.47$ (d, $1 \mathrm{H}, J=9.5 \mathrm{~Hz})$, $2.11(\mathrm{~d}, 1 \mathrm{H}, J=9.5 \mathrm{~Hz}), 1.50$ (s, 9H), 0.84 (s, 9H), 0.02 (s, 3H), -0.02 (s, 3H). ${ }^{13} \mathrm{C}$ NMR (75 MHz, $\left.\mathrm{CDCl}_{3}\right): \_173.6,173.2,149.1,133.8,133.2,132.5,131.5,127.8,127.7,126.9$, $126.7,124.7,124.3,118.2,117.9,83.9,57.4,52.4,39.5,39.3,31.5,27.9,26.9,26.8,25.8$, 24.8, 22.2, 18.2, -5.5, -5.6. IR $\left(\mathrm{CHCl}_{3}\right): 3028,2951,2929,2856,1736,1714,1435,1358$, 1236, 1155, 1080, 839, 756, $702 \mathrm{~cm}^{-1} \cdot \mathrm{m} / z$ (ESI): $680\left(\mathrm{MNa}^{+}, 100 \%\right), 658(72 \%), 602$ (13\%), 558 (27\%). Anal. Calcd. for $\mathrm{C}_{38} \mathrm{H}_{47} \mathrm{NO}_{7} \mathrm{Si}: \mathrm{C}, 69.38 ; \mathrm{H}, 7.20 ; \mathrm{N}, 2.13$. Found: $\mathrm{C}$, 69.17; H, 7.43; N, 2.17. HPLC analysis: 94\% ee (Chiralcel OD-H, $3 \%{ }^{i} \mathrm{PrOH}, 1 \mathrm{ml} / \mathrm{min}$, $=254 \mathrm{~nm}, t_{\mathrm{R}}=5.4 \min$ (minor); $t_{\mathrm{R}}=8.5$ (major)).

\section{Compound (S1)}

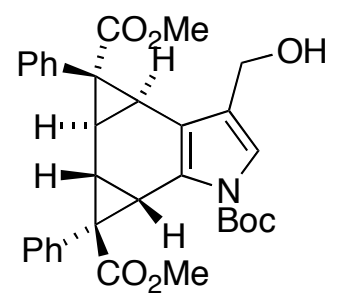

Tetrabutylammonium fluoride (1.0M solution in THF, $0.69 \mathrm{~mL}, 0.69 \mathrm{mmol}, 1.5$ eq.) was added to a solution of ent-31 (302 $\mathrm{mg}, 0.46 \mathrm{mmol}, 1.0$ eq.) in THF $(15 \mathrm{~mL})$ at $0{ }^{\circ} \mathrm{C}$. The reaction mixture as stirred for $3.5 \mathrm{~h} . \mathrm{CH}_{2} \mathrm{Cl}_{2}(20 \mathrm{~mL})$ and brine $(20 \mathrm{~mL})$ were added, and the mixture was shaken and separated. The aqueous layer was extracted with a further portion of $\mathrm{CH}_{2} \mathrm{Cl}_{2}$. The combined organic fractions were dried $\left(\mathrm{MgSO}_{4}\right)$ and concentrated in vacuo. The reside was purified by flash chromatography on silica gel using 1:2 hexanes/diethyl ether as eluant to isolate a white solid, $175 \mathrm{mg}$ (70\%). Mp 109-110 ${ }^{\circ} \mathrm{C}$. $[\alpha]_{\mathrm{D}} 41.6\left(c\right.$ 1.03, $\left.\mathrm{CHCl}_{3}\right) .{ }^{1} \mathrm{H} \mathrm{NMR}\left(\mathrm{CDCl}_{3}, 400 \mathrm{MHz}\right): \delta 7.25-7.15(\mathrm{~m}, 6 \mathrm{H}), 7.10-7.04$ (m, 3H), 7.01-6.91 (m, 2H), 4.40 (d, 1H, $J=13.0 \mathrm{~Hz}), 4.36$ (d, 1H, $J=13.0 \mathrm{~Hz}), 3.59$ (s, $3 \mathrm{H}), 3.58(\mathrm{~s}, 3 \mathrm{H}), 2.75(\mathrm{~d}, 1 \mathrm{H}, J=10.0 \mathrm{~Hz}), 2.71(\mathrm{~d}, 1 \mathrm{H}, J=9.0 \mathrm{~Hz}), 2.52(\mathrm{~d}, 1 \mathrm{H}, J=10$ 
$\mathrm{Hz}), 2.13$ (d, 1H, $J=9.0 \mathrm{~Hz}), 1.51$ (s, 9H). ${ }^{13} \mathrm{C} \mathrm{NMR}\left(\mathrm{CDCl}_{3}, 75 \mathrm{MHz}\right): \delta 173.5,173.1$, $148.9,133.8,133.2,132.2,131.5,127.8,126.9,126.8,125.1,124.3,118.7,117.6,84.2$, 56.8, 52.6, 52.5, 39.6, 39.5, 27.9 (2C), 27.0, 26.9, 24.3. IR $\left(\mathrm{CHCl}_{3}\right): 3510,3027,2950$, 2917, 1708, 1462, 1368, 1242, $796 \mathrm{~cm}^{-1} . m / z$ (ESI): $566\left(\mathrm{MNa}^{+}, 100 \%\right), 544(34 \%), 444$ (26\%), 186 (31\%). Anal Calcd. for $\mathrm{C}_{32} \mathrm{H}_{33} \mathrm{NO}_{7}$ : C, 70.70; H, 6.12; N, 2.58. Found: $\mathrm{C}$, 71.06; H, 6.43; N, 2.48.

\section{Compound (32)}

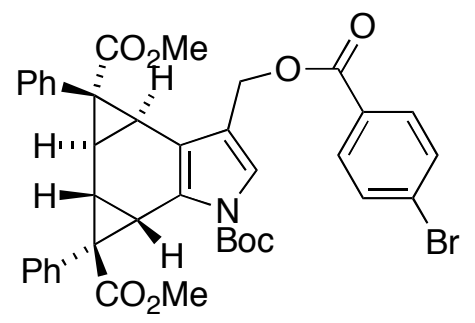

32

Dicyclohexylcarbodiimide (1.0M in $\mathrm{CH}_{2} \mathrm{Cl}_{2}, 114 \mu \mathrm{L}, 114 \mu \mathrm{mol}, 2.0$ eq) was added to a cold $\left(0{ }^{\circ} \mathrm{C}\right)$ solution of compound $\mathbf{S 1}(31 \mathrm{mg}, 57 \mu \mathrm{mol}, 1.0$ eq.), 4-bromobenzoic acid (23 mg, $114 \mu \mathrm{mol}, 2.0$ eq.) and DMAP (3 crystals, catalytic) in $\mathrm{CH}_{2} \mathrm{Cl}_{2}(5 \mathrm{~mL})$. The reaction mixture was stirred for $16 \mathrm{~h}$, allowing to reach room temperature overnight. The mixture was filtered through a plug of cotton, and the filtrate was washed with water. The organic fraction was dried $\left(\mathrm{MgSO}_{4}\right)$ and concentrated in vacuo. The residue was purified by flash chromatography on silica gel using 2:1 hexanes/diethyl ether as eluant to isoalte a white solid, $35 \mathrm{mg}(86 \%)$. Mp 169-170 ${ }^{\circ} \mathrm{C} .[\alpha]_{\mathrm{D}} 30.3\left(c \mathrm{c} 0.15, \mathrm{CHCl}_{3}\right) .{ }^{1} \mathrm{H}$ NMR $\left(\mathrm{CDCl}_{3}, 500\right.$ MHz): $\delta 7.75$ (2H, d, $J=8.5 \mathrm{~Hz}), 7.53$ (d, 2H, $J=8.5 \mathrm{~Hz}), 7.23-7.14(\mathrm{~m}, 6 \mathrm{H}), 7.12-7.03$ (m, $3 \mathrm{H}), 6.99-6.91(\mathrm{~m}, 2 \mathrm{H}), 5.06(\mathrm{~d}, 1 \mathrm{H}, J=12.5 \mathrm{~Hz}), 5.02(\mathrm{~d}, 1 \mathrm{H}, J=12.5 \mathrm{~Hz}), 3.58(\mathrm{~s}, 3 \mathrm{H})$, $3.50(\mathrm{~s}, 3 \mathrm{H}), 2.73(\mathrm{~d}, 2 \mathrm{H}, J=10.0 \mathrm{~Hz}), 2.51(\mathrm{~d}, 1 \mathrm{H}, J=10.0 \mathrm{~Hz}), 2.12(\mathrm{~d}, 1 \mathrm{H}, J=10.0 \mathrm{~Hz})$, $1.51(\mathrm{~s}, 9 \mathrm{H})$. Due to the small amount of product obtained, and the high molecular weight 
of this compound, a ${ }^{13} \mathrm{C}$ NMR spectrum was not obtained. IR $\left(\mathrm{CHCl}_{3}\right)$ : 3028, 2982, 2953, $1716,1591,1433,1371,1256,1238,1155,1099,733,702 \mathrm{~cm}^{-1}$. The identity of this compound is confirmed by x-ray crystallography (page S42) and the purity by the ${ }^{1} \mathrm{H}$ NMR spectrum (page S33).

tert-Butyl 3-((1R,2R)-1-(tert-butyldimethylsilyloxy)-3-((S)-1-ethoxy-1-oxopropan-2yloxy)-3-0xo-2-phenylpropyl)-1 $H$-indole-1-carboxylate (34)

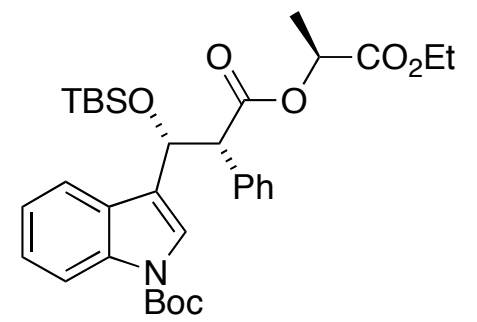

34

A solution of compound 28 (147 mg, $0.41 \mathrm{mmol}, 1.00$ eq.), $\mathrm{Rh}_{2}(\mathrm{OOct})_{4}(6.3 \mathrm{mg}, 0.008$ mmol, 0.02 eq.) and 2,2-dimethylbutane (5 mL) was degassed with argon for $10 \mathrm{~min}$. A solution of compound $\mathbf{3 3}^{8}$ (213 mg, $0.81 \mathrm{mmol}, 2.00$ eq.) in 2,2-dimethylbutane (9 mL) was degassed with argon for $10 \mathrm{~min}$ and then added to the reaction mixture using a syringe pump over $3 \mathrm{~h}$. The reaction mixture was stirred for $1 \mathrm{~h}$, and then concentrated in vacuo. Crude NMR showed 6.4:1 syn/anti. Purified by flash chromatography on silica gel using 20:1 hexane $/ \mathrm{Et}_{2} \mathrm{O}$ as eluant to isolate a mixture of all the anti and syn isomers as a pale yellow oil, $103 \mathrm{mg}(42 \%) .{ }^{1} \mathrm{H}$ NMR $\left(500 \mathrm{MHz}, \mathrm{CDCl}_{3}\right)$ :_8.16-7.99 (m, 1H), 7.81 (d, $1 \mathrm{H}, J=7.5 \mathrm{~Hz}), 7.52-7.44(\mathrm{~m}, 3 \mathrm{H}), 7.36-7.22(\mathrm{~m}, 5 \mathrm{H}), 5.40(\mathrm{~d}, 1 \mathrm{H}, J=9.5 \mathrm{~Hz}), 4.82(\mathrm{q}$, 1H, J=7.0 Hz), $4.20(\mathrm{~d}, 1 \mathrm{H}, J=9.5 \mathrm{~Hz}), 4.06-3.98(\mathrm{~m}, 2 \mathrm{H}), 1.68(\mathrm{~s}, 9 \mathrm{H}), 1.12(\mathrm{~d}, 3 \mathrm{H}$, $J=7.0 \mathrm{~Hz}), 1.06(\mathrm{t}, 3 \mathrm{H}, J=7.0 \mathrm{~Hz}), 0.61(\mathrm{~s}, 9 \mathrm{H}),-0.33(\mathrm{~s}, 3 \mathrm{H}),-0.37(\mathrm{~s}, 3 \mathrm{H})$. The tert-butyl signals of the TBS group for the other diastereomers come into resonance at_0.85 (anti), _0.84 (anti),_0.60 (syn). ${ }^{13} \mathrm{C}$ NMR (75 MHz, $\left.\mathrm{CDCl}_{3}\right): \delta 171.0,170.2,149.7,135.9,135.7$, $129.5,128.5,128.0,127.4,124.3,123.8,122.4,122.1,121.0,115.0,83.6,71.1,68.7$, 
61.0, 59.4, 28.2, 25.4, 17.8, 16.4, 13.8, -5.1, -5.9. IR $\left(\mathrm{CHCl}_{3}\right): 2978,2956,2929,2856$, 1736, 1452, 1369, 1254, 1155, 1088, 835, 777, $698 \mathrm{~cm}^{-1} . \mathrm{m} / z$ (ESI): $618\left(\mathrm{MNa}^{+}, 100 \%\right)$, $613(25 \%), 464$ (11\%). HRMS (ESI) Calcd. for $\mathrm{C}_{33} \mathrm{H}_{45} \mathrm{O}_{7} \mathrm{NNaSi}\left(\mathrm{MNa}^{+}\right)$: 618.2858 . Found: 618.2850 .

tert-Butyl 3-((1R,2S)-1-(tert-butyldimethylsilyloxy)-3-hydroxy-2-phenylpropyl)-1 Hindole-1-carboxylate (35)

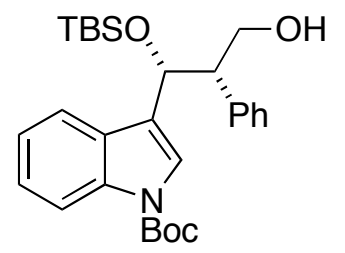

35

Diisobutyl aluminium hydride (1.0 M in toluene, $0.52 \mathrm{~mL}, 0.52 \mathrm{mmol}, 4$ eq.) was added dropwise to a cold $\left(0{ }^{\circ} \mathrm{C}\right)$ solution of compound $34(66 \mathrm{mg}, 0.13 \mathrm{mmol}, 1$ eq. $)$. The reaction mixture was stirred for $1 \mathrm{~h}$, after which time TLC indicates disappearance of the starting material. Poured into 1:1 diethyl ether/saturated aqueous potassium sodium tartrate and stirred for $45 \mathrm{~min}$. The layers were separated and the aqueous layer further extracted with diethyl ether $(2 \mathrm{x})$. The combined organic fractions were dried $\left(\mathrm{Na}_{2} \mathrm{SO}_{4}\right)$ and concentrated in vacuo. Purified by flash chromatography on silica gel using 4:1 petroleum ether/diethyl ether as eluant. Isolated product $\mathbf{3 4}$ as a pale yellow oil, $31 \mathrm{mg}$ (50\%). Mp 45-47 ${ }^{\circ} \mathrm{C} .[\alpha]_{\mathrm{D}} 42.9\left(72 \%\right.$ ee) $\left(c \mathrm{c} 0.35, \mathrm{CHCl}_{3}\right) .{ }^{1} \mathrm{H} \mathrm{NMR}\left(\mathrm{CDCl}_{3}, 400 \mathrm{MHz}\right)$ : $\delta 8.12$ (br s, 1H), $7.44(\mathrm{~d}, 1 \mathrm{H}, J=7.5 \mathrm{~Hz}), 7.36-7.21(\mathrm{~m}, 4 \mathrm{H}), 7.19-7.12(\mathrm{~m}, 3 \mathrm{H}), 5.26$ (d, $1 \mathrm{H}, J=5.5 \mathrm{~Hz}), 4.01-3.82(\mathrm{~m}, 2 \mathrm{H}), 3.45-3.36(\mathrm{~m}, 1 \mathrm{H}), 1.81(\mathrm{br} \mathrm{s}, 1 \mathrm{H}), 1.68(\mathrm{~s}, 9 \mathrm{H}), 0.81$ (s, 9H), -0.07 (s, 3H), -0.23 (s, 3H). ${ }^{13} \mathrm{C}$ NMR ( $\left.\mathrm{CDCl}_{3}, 75 \mathrm{MHz}\right): 8149.7,139.3,135.5$, 129.3, 128.7, 128.1, 126.9, 124.2, 123.4, 122.3, 120.4, 115.0, 83.6, 71.0, 63.8, 55.1, 29.7, 28.2, 25.6, 18.0, -4.9, -5.6. IR $\left(\mathrm{CHCl}_{3}\right): 3460,2953,2929,2856,1734,1452,1371,1254$, 
1159, 1090, 1070, 837, 775, 746, $700 \mathrm{~cm}^{-1} . \mathrm{m} / z$ (ESI): $504\left(\mathrm{MNa}^{+}, 13 \%\right), 350(100 \%)$, 294 (19\%), 250 (11\%). HRMS (ESI) Calcd. for $\mathrm{C}_{28} \mathrm{H}_{39} \mathrm{O}_{4} \mathrm{NNaSi}\left(\mathrm{MNa}^{+}\right)$: 504.2541. Found: 504.2523. HPLC analysis $72 \%$ ee (R,R-Whelk-O1, $3 \%{ }^{i} \mathrm{PrOH}, 1 \mathrm{ml} / \mathrm{min},{ }_{-}=254$ $\mathrm{nm}, t_{\mathrm{R}}=10.2 \min \left(\right.$ minor); $t_{\mathrm{R}}=14.8$ (major)).

tert-Butyl 5-bromo-3-(hydroxymethyl)-1H-indole-1-carboxylate

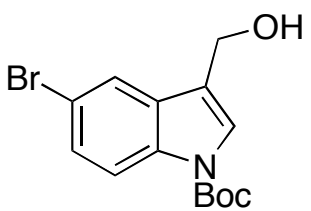

Sodium borohydride (320 mg, $8.46 \mathrm{mmol}, 2$ eq.) was added to a suspension of tert-butyl 5-bromo-3-formyl-1H-indole-1-carboxylate ${ }^{9}(1.37 \mathrm{~g}, 4.23 \mathrm{mmol}, 1$ eq.) in ethanol (25 $\mathrm{mL}$ ), and became a homogeneous solution after $10 \mathrm{~min}$. The reaction mixture was stirred for $6 \mathrm{~h}$, and then concentrated in vacuo. The residue was partitioned between $\mathrm{Et}_{2} \mathrm{O}$ and aqueous $1 \mathrm{M} \mathrm{NaOH}$ solution, shaken and separated. The aqueous fraction was extracted with a further two portions $\mathrm{Et}_{2} \mathrm{O}$. The combined organic extracts were dried over $\mathrm{MgSO}_{4}$ and then concentrated in vacuo to a white solid, $1.37 \mathrm{~g}(99 \%)$. Mp $105-107{ }^{\circ} \mathrm{C} .{ }^{1} \mathrm{H}$ NMR (500 MHz, $\mathrm{CDCl}_{3}$ ):_7.92-7.83 (br m, 1H), 7.65 (d, 1H, J=1.5 Hz), 7.45 (s, 1H), 7.30 (dd, $1 \mathrm{H}, J=9.0,1.5 \mathrm{~Hz}), 4.82$ (s, 2H), 3.41 (br s, $1 \mathrm{H}), 1.67$ (s, 9H). ${ }^{13} \mathrm{C} \mathrm{NMR}(75 \mathrm{MHz}$, $\left.\mathrm{CDCl}_{3}\right):$ 8149.1, 134.1, 130.6, 127.0, 124.3, 121.8, 119.6, 116.3, 115.7, 84.0, 56.3, 27.9. IR ( $\left.\mathrm{CHCl}_{3}\right): 3385,2980,2931,2874,1732,1446,1369,1275,1255,1153,1095,1058$, $729 \mathrm{~cm}^{-1} . \mathrm{m} / z$ (EI): $327\left(\mathrm{M}^{81} \mathrm{Br}^{+}, 27 \%\right), 325\left(\mathrm{M}^{79} \mathrm{Br}^{+}, 27 \%\right), 271$ (56\%), 269 (56\%), 210 (42\%), 208 (42\%), 117 (48\%), 57 (100\%). Anal. Calcd. for $\mathrm{C}_{14} \mathrm{H}_{16} \mathrm{BrO}_{3} \mathrm{~N}$ : C, 51.55; H, 4.94; N, 4.29. Found: C, 51.66; H, 4.80; N, 4.35. 
tert-Butyl 5-bromo-3-((tert-butyldimethylsilyloxy)methyl)-1H-indole-1-carboxylate (36)

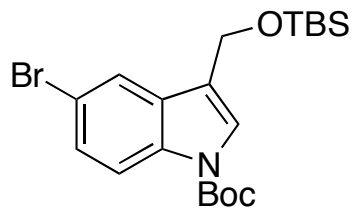

36

A solution of 5-bromo-3-hydroxymethyl-indole-1-carboxylic acid tert-butyl ester (1.37 g, $4.18 \mathrm{mmol}, 1.0$ eq.) and tert-butyldimethylsilyl chloride (693 mg, $4.60 \mathrm{mmol}, 1.1$ eq.) in dichloromethane $(30 \mathrm{~mL})$ was stirred until homogeneous, and imidazole $(341 \mathrm{mg}, 5.01$ mmol, 1.2 eq.) was added. A white precipitate formed immediately, and was stirred for 6 h. The reaction mixture was filtered through a plug of silica, and then concentrated in vacuo. The residue was purified by flash chromatography using $20: 1$ hexane $/ \mathrm{Et}_{2} \mathrm{O}$ to provide a white solid, $1.39 \mathrm{~g}(76 \%) \mathrm{mp} 50-52{ }^{\circ} \mathrm{C} .{ }^{1} \mathrm{H}$ NMR $\left(500 \mathrm{MHz}, \mathrm{CDCl}_{3}\right)$ :_8.01 (br, 1H), $7.76(\mathrm{~d}, 1 \mathrm{H}, J=2.0 \mathrm{~Hz}), 7.51(\mathrm{~s}, 1 \mathrm{H}), 7.41(\mathrm{dd}, 1 \mathrm{H}, J=9.0,2.0 \mathrm{~Hz}), 4.83(\mathrm{~s}, 2 \mathrm{H})$, 1.67 (s, 9H), 0.96 (s, 9H), $0.14(\mathrm{~s}, 6 \mathrm{H}) .{ }^{13} \mathrm{C} \mathrm{NMR}\left(\mathrm{CDCl}_{3}, 75 \mathrm{MHz}\right): \delta 149.4,134.5$, $130.9,127.1,123.9,122.3,120.4,116.6,115.8,83.9,57.8,28.1,25.9,18.3,-5.3$. IR $\left(\mathrm{CHCl}_{3}\right): 2955,2929,2856,1736,1448,1369,1255,1155,1055,837,775 \mathrm{~cm}^{-1} . \mathrm{m} / z(\mathrm{EI}):$ $441\left(\mathrm{M}^{81} \mathrm{Br}^{+}, 11 \%\right), 439\left(\mathrm{M}^{79} \mathrm{Br}^{+}, 11 \%\right), 384(16 \%), 382$ (16\%), $328(56 \%), 326$ (56\%), 284 (56\%), 282 (57\%), 75 (76\%), 57 (100\%). Anal. Calcd. for $\mathrm{C}_{20} \mathrm{H}_{30} \mathrm{BrNO}_{3} \mathrm{Si}: \mathrm{C}, 54.54$; H, 6.87; N, 3.18. Found: C, 54.60; H, 7.08; N, 3.02. 
tert-Butyl 5-bromo-3-((1S,2S)-1-(tert-butyldimethylsilyloxy)-3-methoxy-3-oxo-2phenylpropyl)-1H-indole-1-carboxylate (37) derived from $\mathrm{Rh}_{2}(S \text {-DOSP })_{4}$ and tertButyl 5-bromo-3-((1R,2R)-1-(tert-butyldimethylsilyloxy)-3-methoxy-3-oxo-2phenylpropyl)-1H-indole-1-carboxylate (ent-37) derived from $\operatorname{Rh}_{2}(S-P T T L)_{4}$

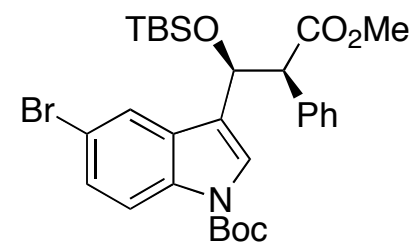

37

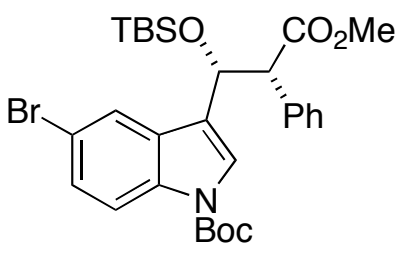

ent-37

Procedure A: A solution of methyl phenyldiazoacetate 29 (125 mg, $0.71 \mathrm{mmol}, 2.00$ mmol) in 2,2-dimethylbutane $(5 \mathrm{~mL})$ was added via a syringe pump over $2 \mathrm{~h}$ to a solution of compound 36 (155 mg, $0.35 \mathrm{mmol}, 1.00$ eq.), $\mathrm{Rh}_{2}(S \text {-DOSP })_{4}(6.7 \mathrm{mg}, 0.007 \mathrm{mmol}$, 0.01 eq.) and 2,2-dimethylbutane $(5 \mathrm{~mL})$ heated under reflux. The reaction mixture was stirred at $50{ }^{\circ} \mathrm{C}$ for $1 \mathrm{~h}$, and then allowed to cool to room temperature and concentrated in vacuo. Crude NMR showed 69\% de syn/anti. Purified by flash chromatography on silica gel using 20:1 hexane/ $\mathrm{Et}_{2} \mathrm{O}$ as eluant to isolate the anti isomer as a colorless oil, $7 \mathrm{mg}$, a mixture of the anti and syn isomers as a colorless oil, $51 \mathrm{mg}$, and the syn isomer as a colorless oil, $94 \mathrm{mg}$. Total mass: $152 \mathrm{mg}$ (74\%).

Procedure A: A solution of methyl phenyldiazoacetate 29 (121 mg, $0.68 \mathrm{mmol}, 2.00$ mmol) in 2,2-dimethylbutane $(5 \mathrm{~mL})$ was added to the reaction mixture via a syringe pump over $2 \mathrm{~h}$ to a solution of compound 36 (150 mg, $0.34 \mathrm{mmol}, 1.00$ eq.), $\mathrm{Rh}_{2}(\mathrm{OOct})_{4}$ (5.3 mg, $0.007 \mathrm{mmol}, 0.01$ eq.) and 2,2-dimethylbutane $(5 \mathrm{~mL})$ heated under reflux. The reaction mixture was stirred at $50{ }^{\circ} \mathrm{C}$ for $1 \mathrm{~h}$, and then allowed to cool to room temperature and concentrated in vacuo. Crude NMR showed $77 \%$ de syn/anti. Purified by flash chromatography on silica gel using 20:1 hexane/Et $\mathrm{t}_{2} \mathrm{O}$ as eluant to isolate a mixture of the anti and syn isomers as a colorless oil, $61 \mathrm{mg}$, and the syn isomer as a colorless oil, $60 \mathrm{mg}$. Total mass: $121 \mathrm{mg}(60 \%)$.

Procedure E: A solution of methyl phenyldiazoacetate 29 (150 mg, $0.85 \mathrm{mmol}, 2.00$ mmol) in 2,2-dimethylbutane $(5 \mathrm{~mL})$ was added via a syringe pump over $2 \mathrm{~h}$ to a solution of compound 36 (187 mg, $0.43 \mathrm{mmol}, 1.00$ eq.), $\mathrm{Rh}_{2}(S$-PTTL) 4 (5.3 mg, $0.004 \mathrm{mmol}$, 0.01 eq.) and 2,2-dimethylbutane $(5 \mathrm{~mL})$ heated under reflux. The reaction mixture was stirred at $50{ }^{\circ} \mathrm{C}$ for $1 \mathrm{~h}$, and then allowed to cool to room temperature and concentrated in vacuo. Crude NMR showed $61 \%$ de syn/anti. Purified by flash chromatography on silica 
gel using 20:1 hexane $/ \mathrm{Et}_{2} \mathrm{O}$ as eluant to isolate the anti isomer as a colorless oil, $4 \mathrm{mg}$, a mixture of the anti and syn isomers as a colorless oil, $26 \mathrm{mg}$, and the syn isomer as a white soild, $143 \mathrm{mg}$. Total mass: $173 \mathrm{mg}(68 \%)$.

anti isomer: ${ }^{1} \mathrm{H}$ NMR $\left(500 \mathrm{MHz}, \mathrm{CDCl}_{3}\right)$ :_7.94-7.86 (m, $\left.1 \mathrm{H}\right), 7.72(\mathrm{~s}, 1 \mathrm{H}), 7.33(\mathrm{dd}, 1 \mathrm{H}$, $J=8.5,1.5 \mathrm{~Hz}), 7.18-7.09(\mathrm{~m}, 5 \mathrm{H}), 7.00(\mathrm{~s}, 1 \mathrm{H}), 5.33$ (d, 1H, $J=10.5 \mathrm{~Hz}), 4.08(\mathrm{~d}, 1 \mathrm{H}$, $J=10.5 \mathrm{~Hz}$ ), 3.76 (s, 3H), 1.60 (s, 9H), 0.87 (s, 9H), 0.12 (s, 3H), -0.25 (s, 3H). syn isomer of ent-37: $[\alpha]_{\mathrm{D}} 56.9\left(94 \%\right.$ ee) $\left(c\right.$ 1.42, $\left.\mathrm{CHCl}_{3}\right) .{ }^{1} \mathrm{H} \mathrm{NMR}\left(500 \mathrm{MHz}, \mathrm{CDCl}_{3}\right)$ : _8.06-7.98 (m, 1H), 7.90 (d, 1H, J=1.5 Hz), 7.49-7.42 (m, 2H), 7.39 (dd, 1H, J=9.0, 1.5 $\mathrm{Hz}), 7.37-7.28$ (m, 5H), 5.38 (d, 1H, J=9.0 Hz), 4.04 (d, 1H, J=9.0 Hz), 3.49 (s, 3H), 1.67 (s, 9H), 0.63 (s, 9H), -0.32 (s, 3H), -0.36 (s, 3H). ${ }^{13} \mathrm{C} \mathrm{NMR}\left(75 \mathrm{MHz}, \mathrm{CDCl}_{3}\right)$ : 8172.0 , 149.3, 136.0, 134.3, 130.1, 129.4, 128.2, 127.6, 127.1, 124.5, 123.6, 121.6, 116.6, 115.8, 84.2, 70.6, 59.9, 51.8, 28.1, 25.4, 17.8, -5.1, -5.9. IR $\left(\mathrm{CHCl}_{3}\right): 2953,2931,2833,2856$, $1738,1450,1383,1371,1352,1257,1155,1099,839,777 \mathrm{~cm}^{-1} . m / z$ (ESI): 612 $\left(\mathrm{M}^{81} \mathrm{BrNa}^{+}, 100 \%\right), 610\left(\mathrm{M}^{79} \mathrm{BrNa}^{+}, 91 \%\right), 557$ (12\%), 402 (16\%), 400 (19\%). HRMS Calcd. for $\mathrm{C}_{29} \mathrm{H}_{38} \mathrm{O}_{5} \mathrm{NBrNaSi}\left(\mathrm{MNa}^{+}\right)$. Found: 610.1607. HPLC analysis $94 \%$ ee (Chiralcel OD-H, $1 \%{ }^{i} \mathrm{PrOH}, 1 \mathrm{ml} / \mathrm{min},{ }_{-}=254 \mathrm{~nm}, t_{\mathrm{R}}=4.6 \mathrm{~min}$ (minor); $t_{\mathrm{R}}=5.3$ (major)). 
Important NOE Interactions to determine the stereochemistry of (10), (11), (17a) and $(17 b)$
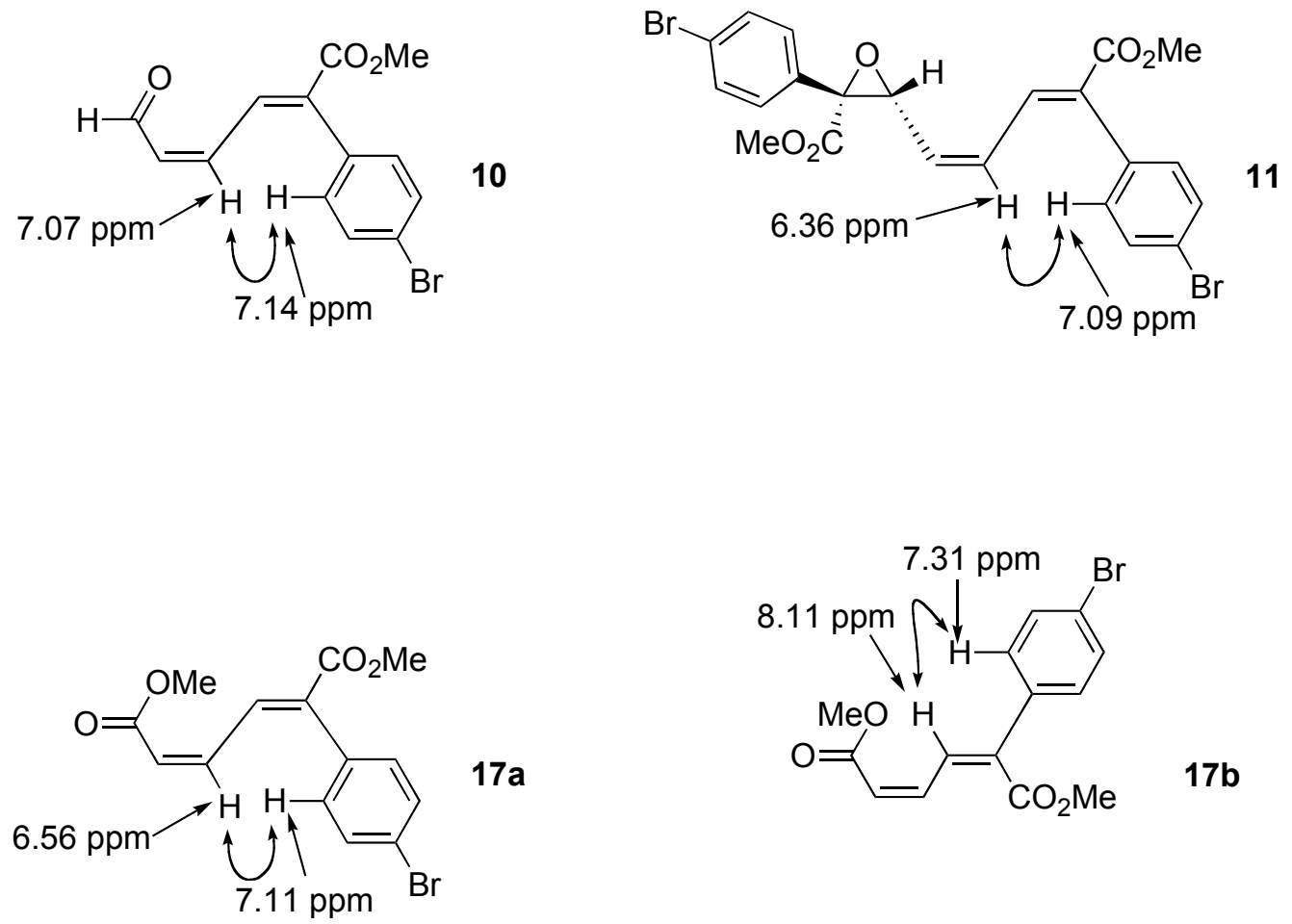


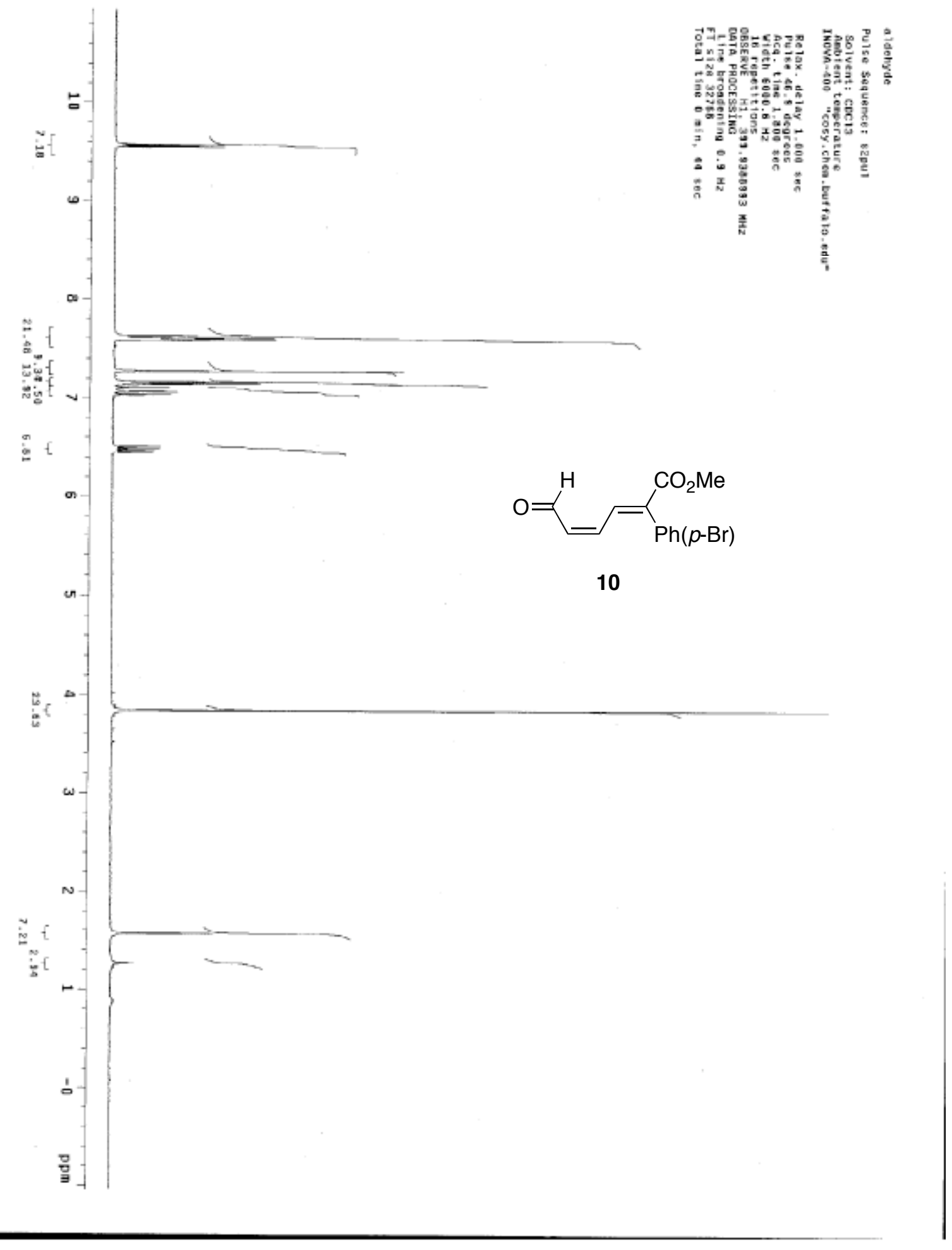




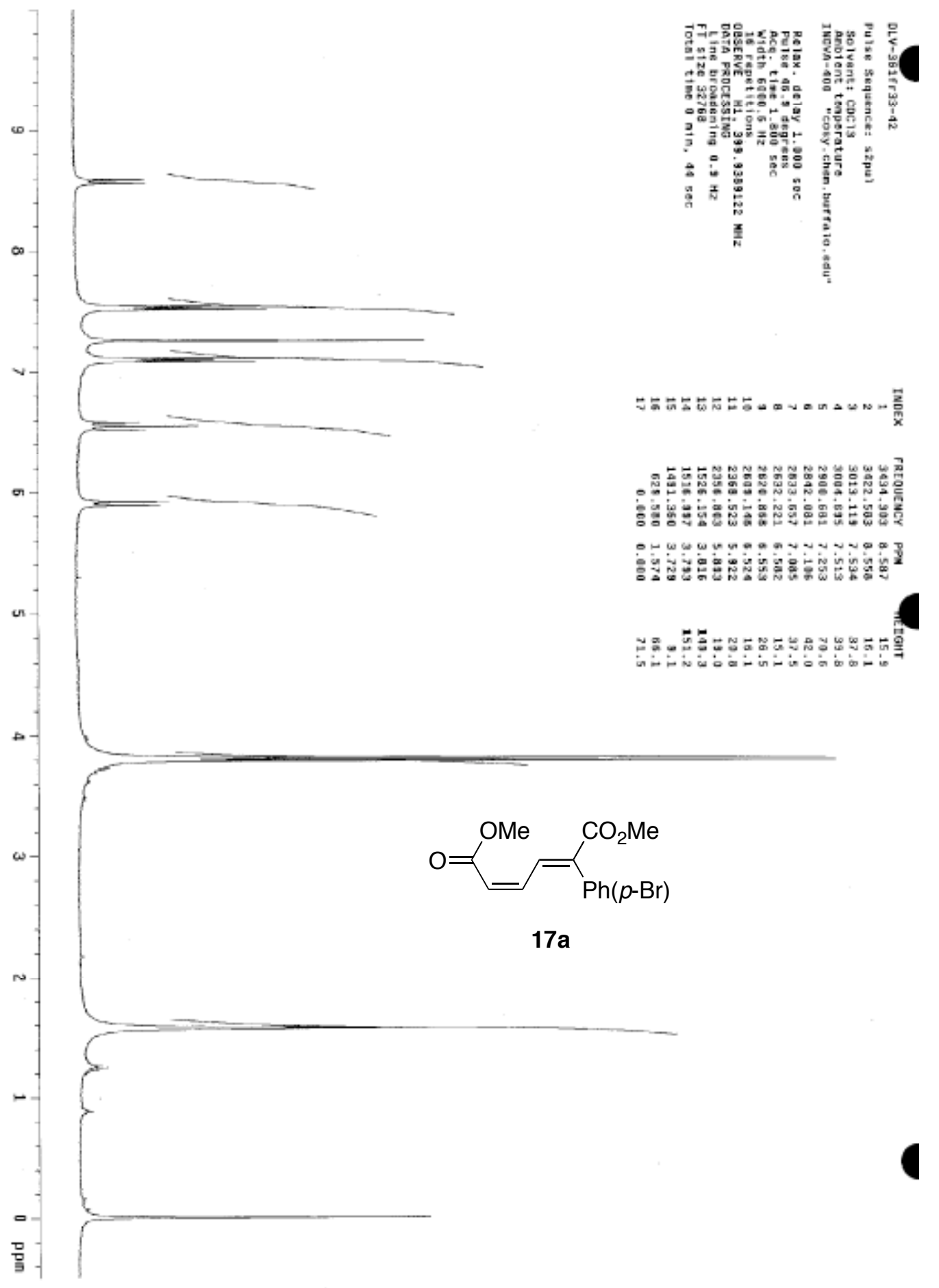




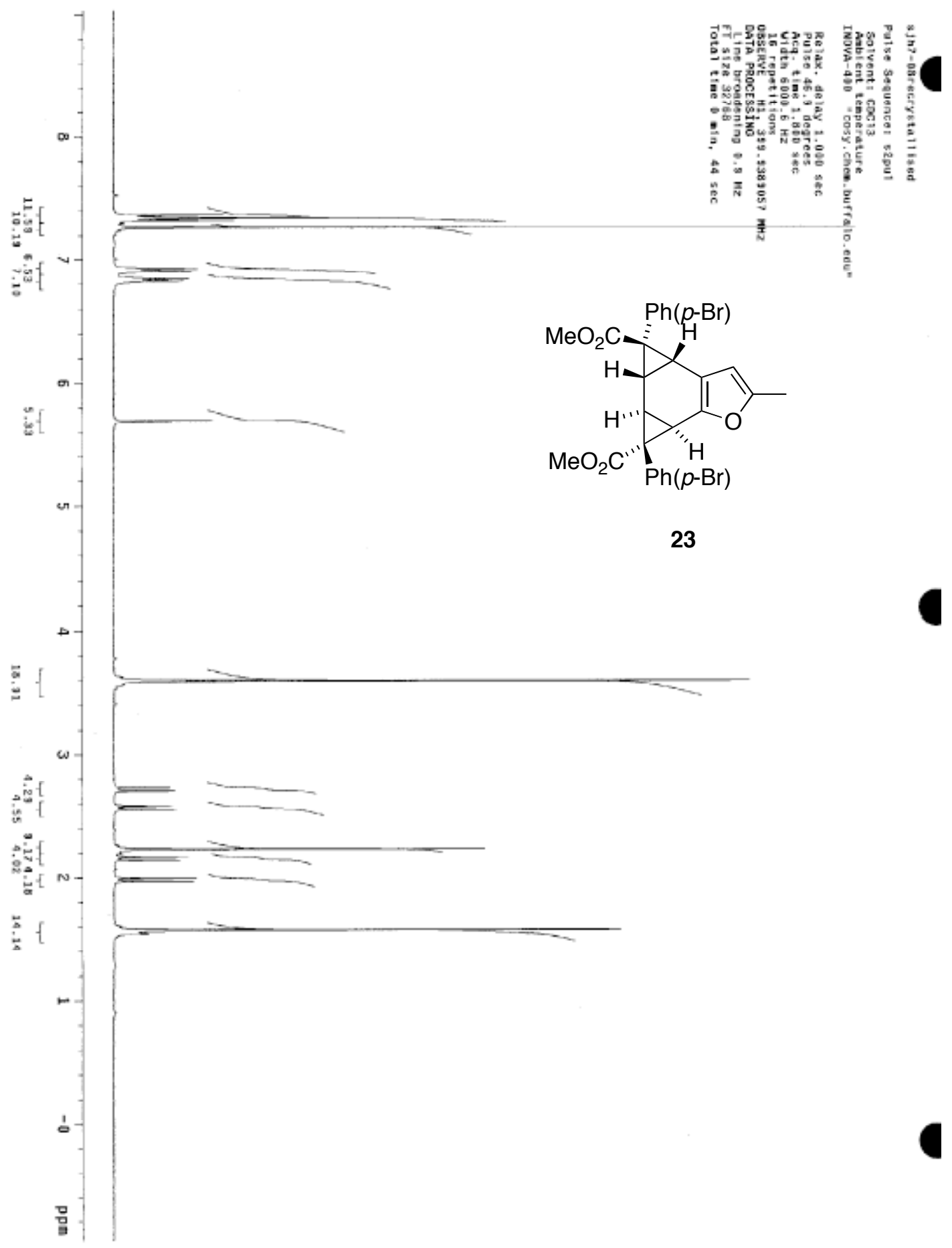




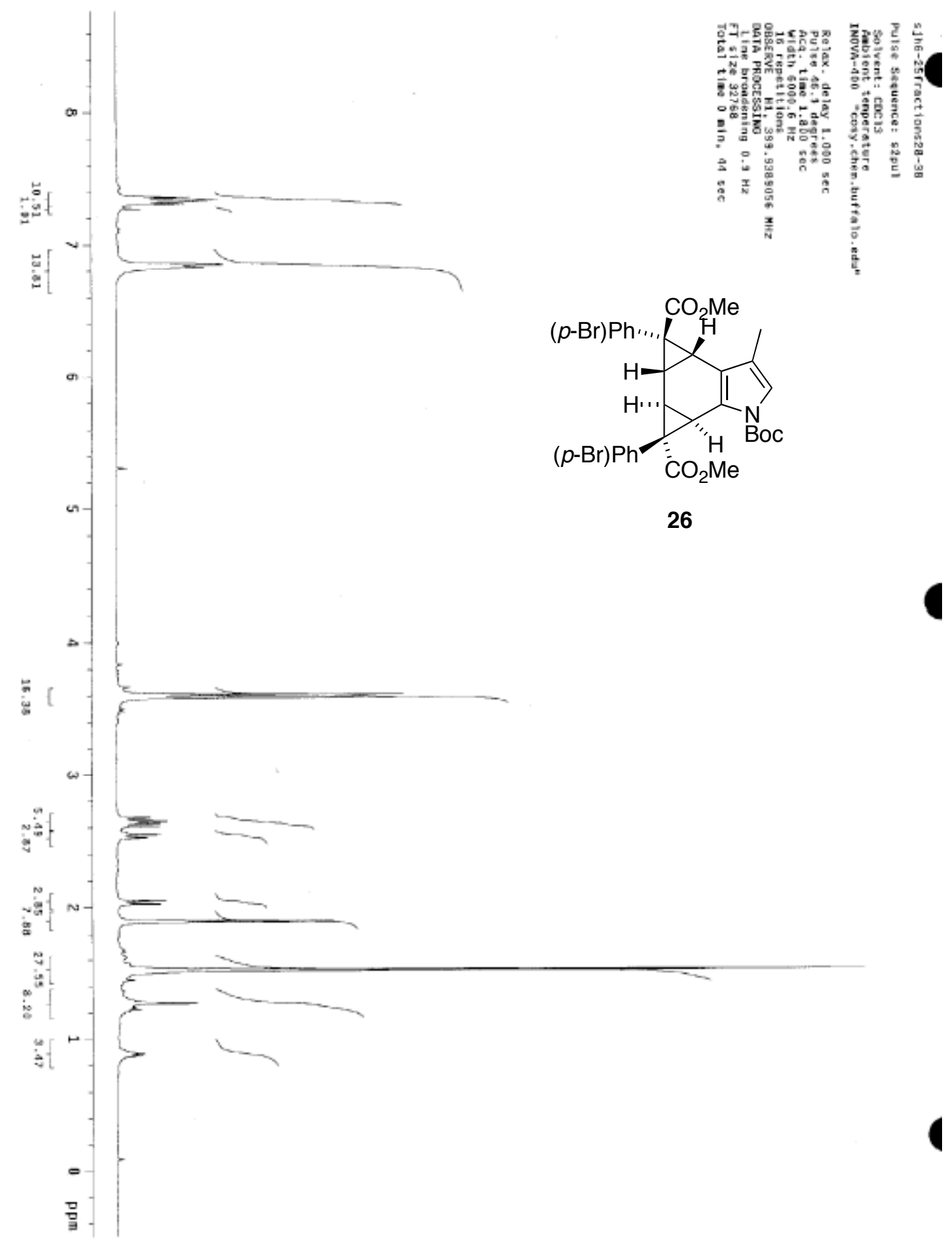




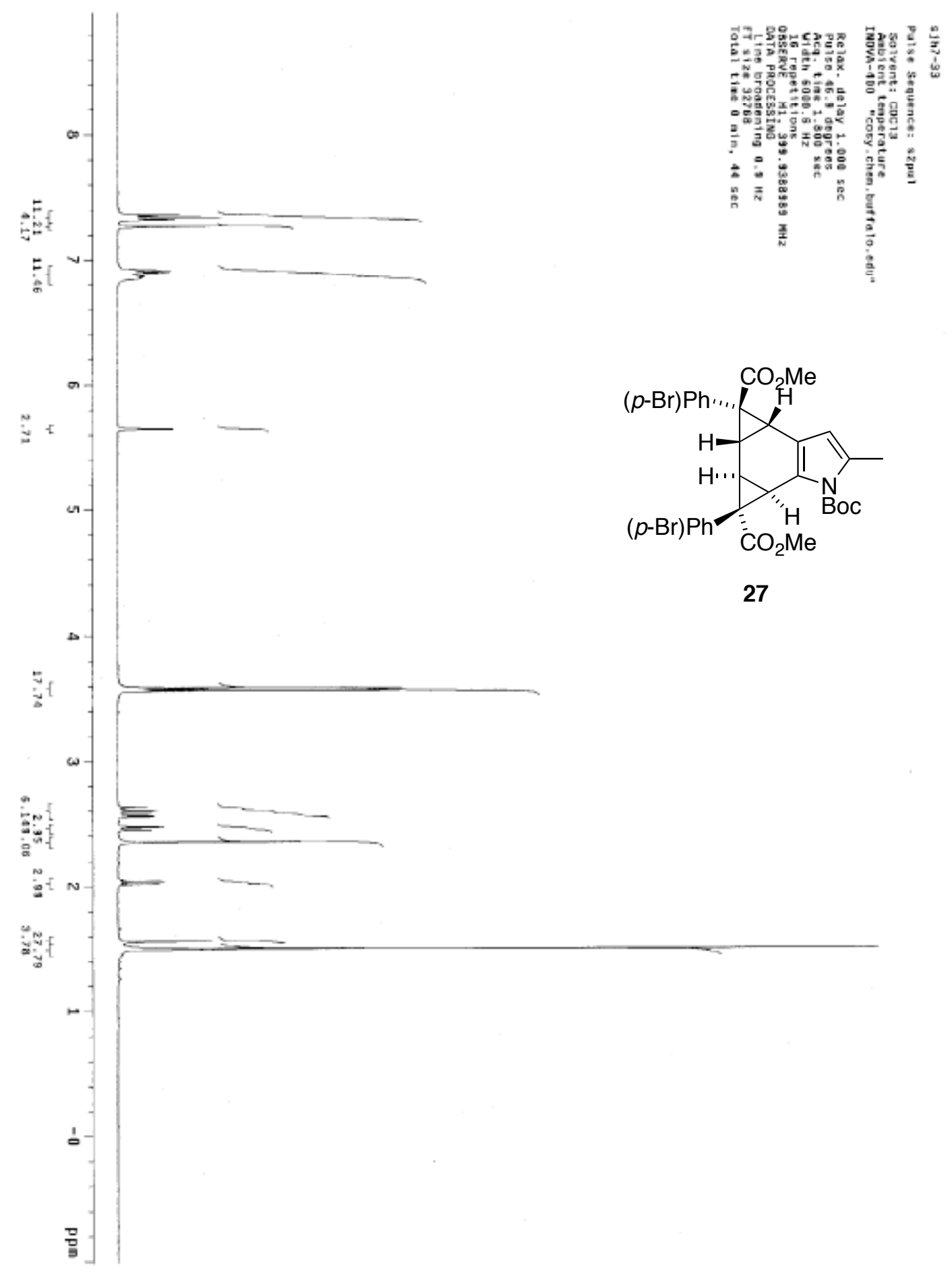




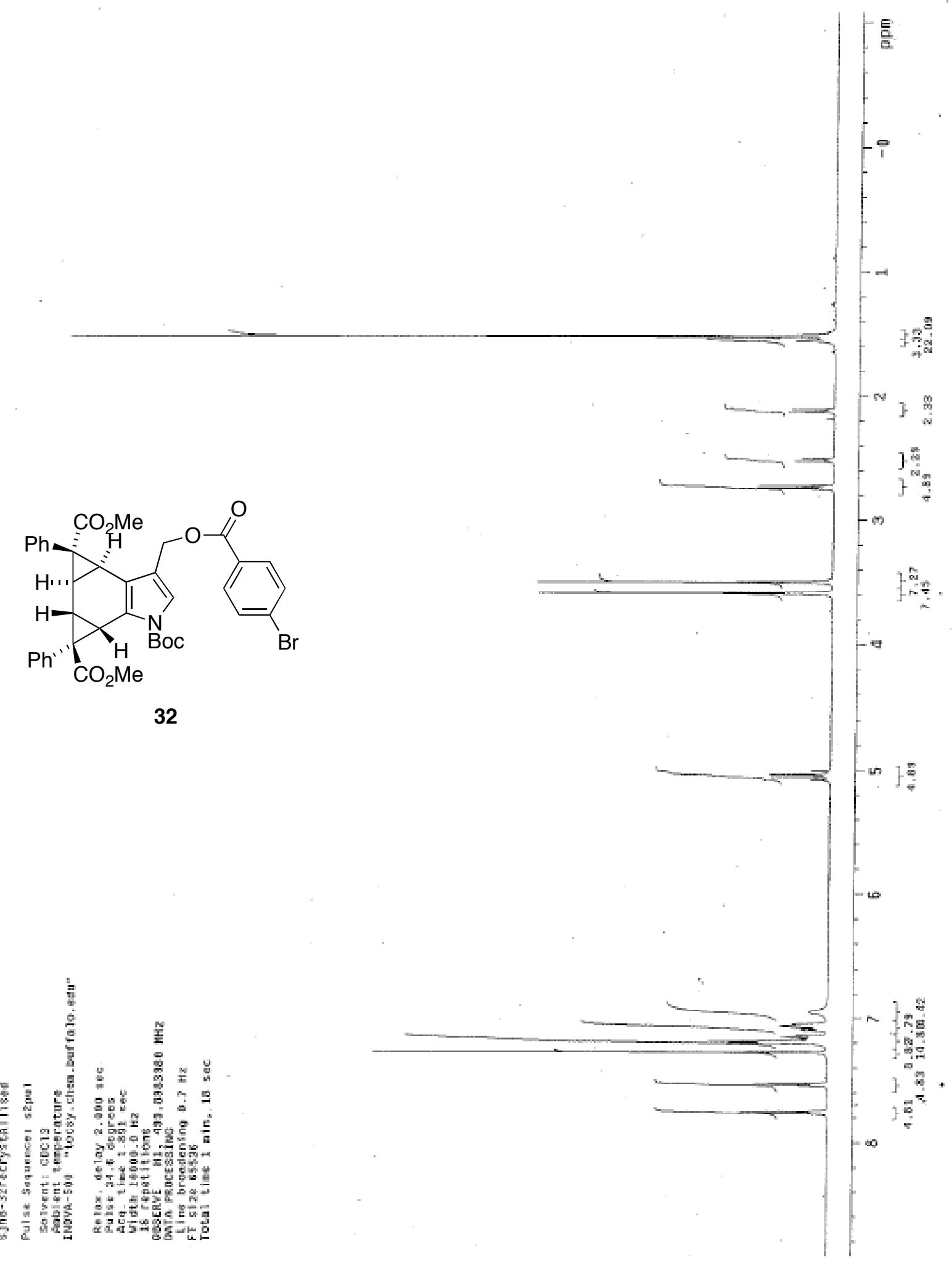




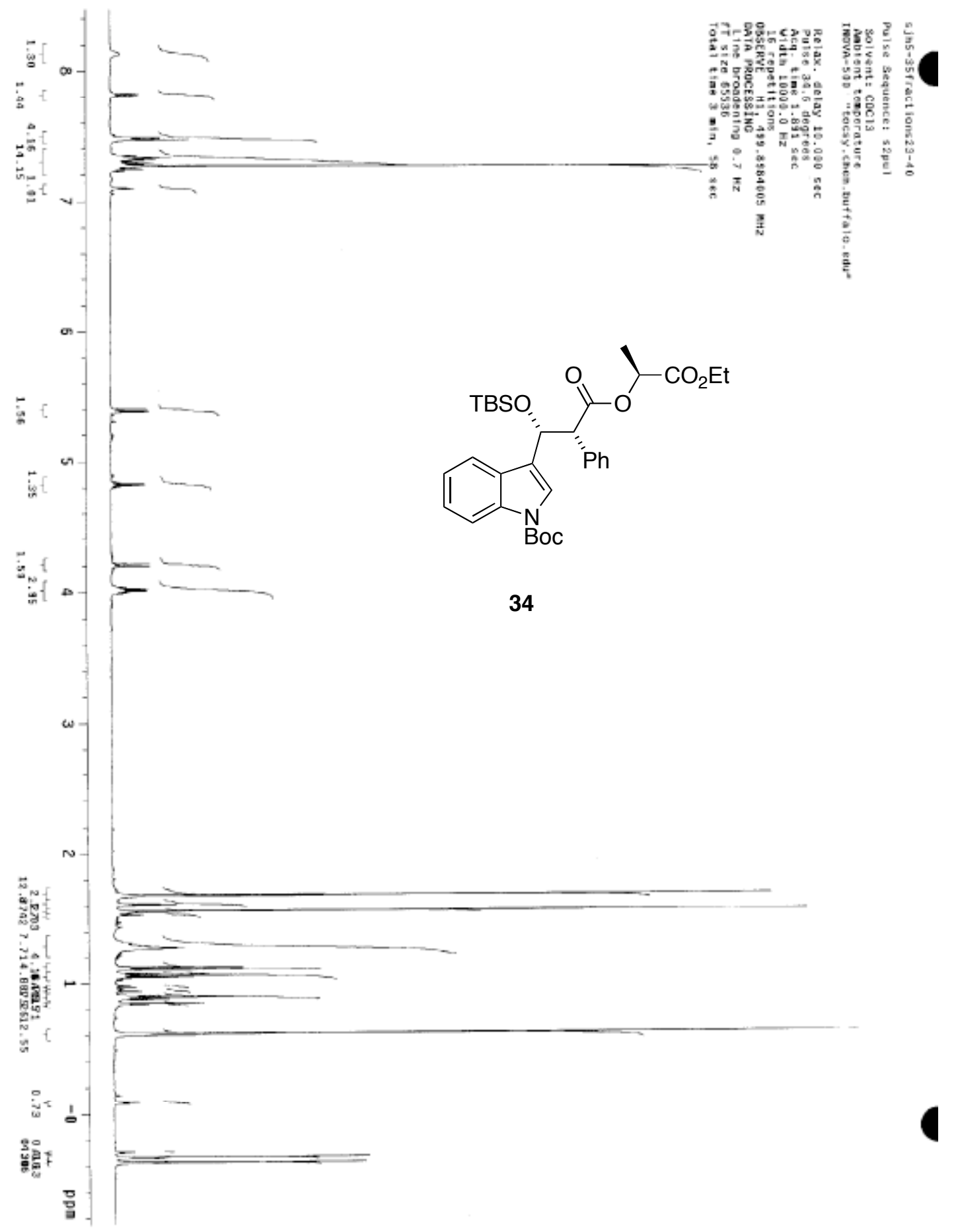




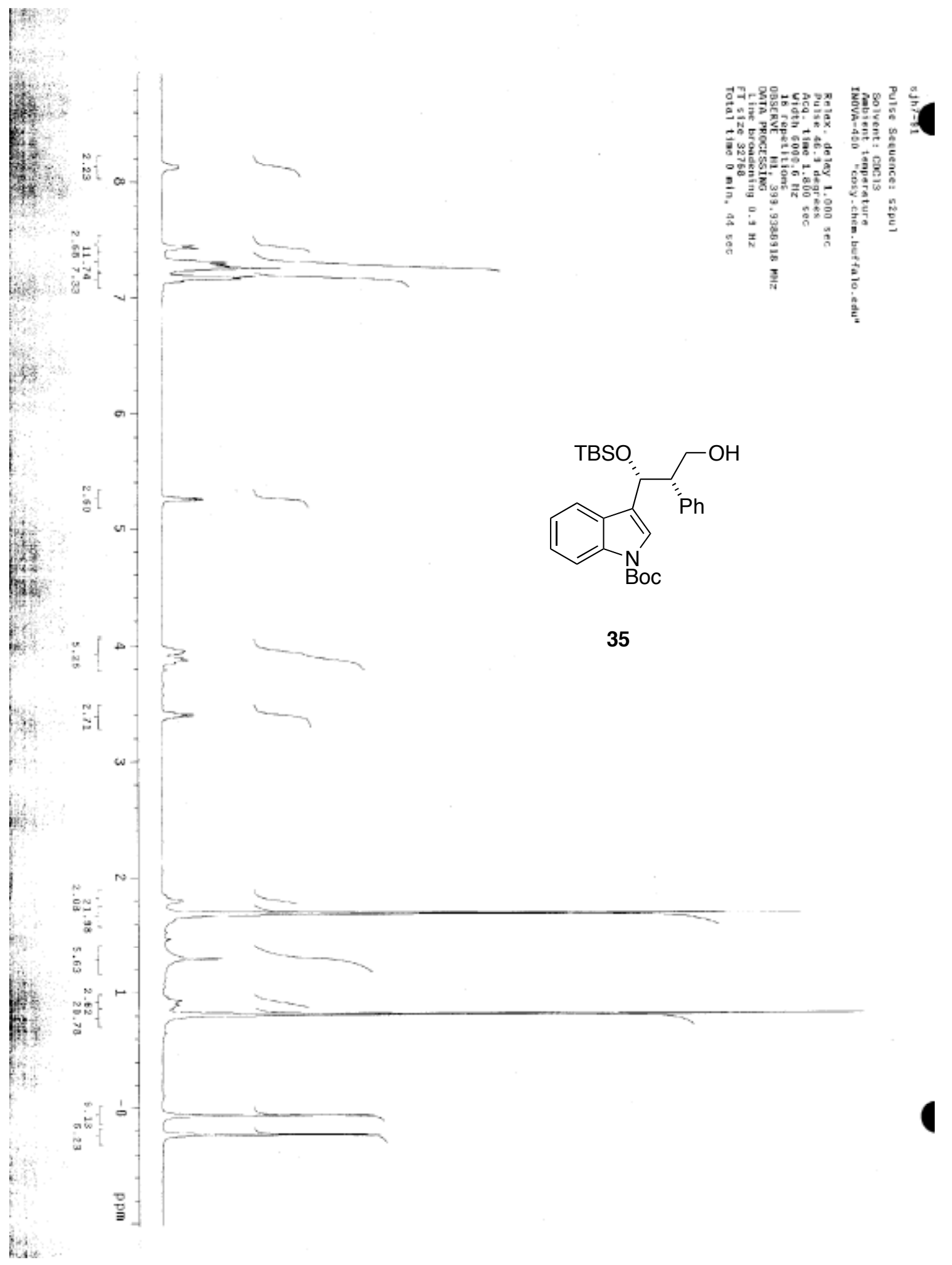




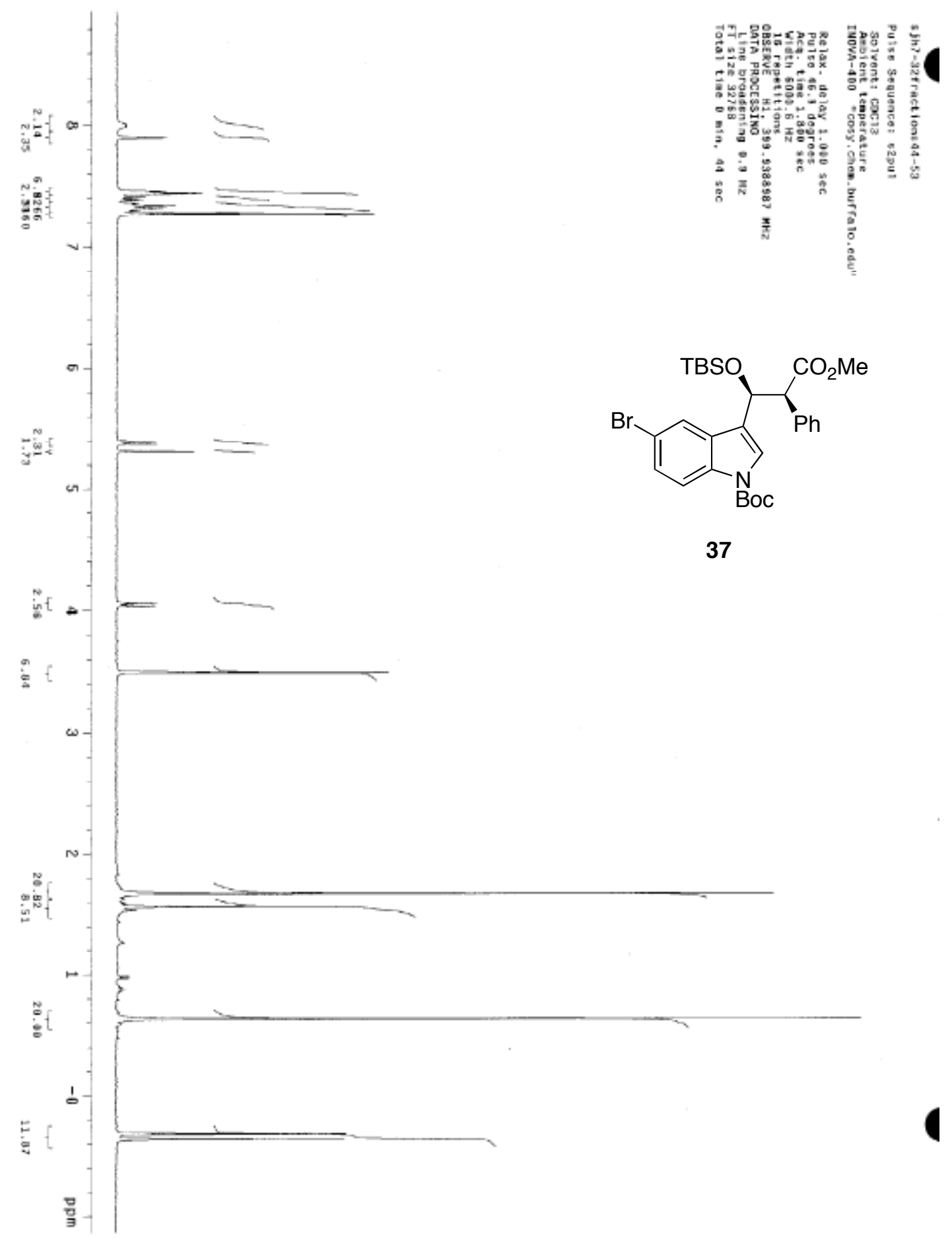




\section{X-ray Crystal Structure of 5}

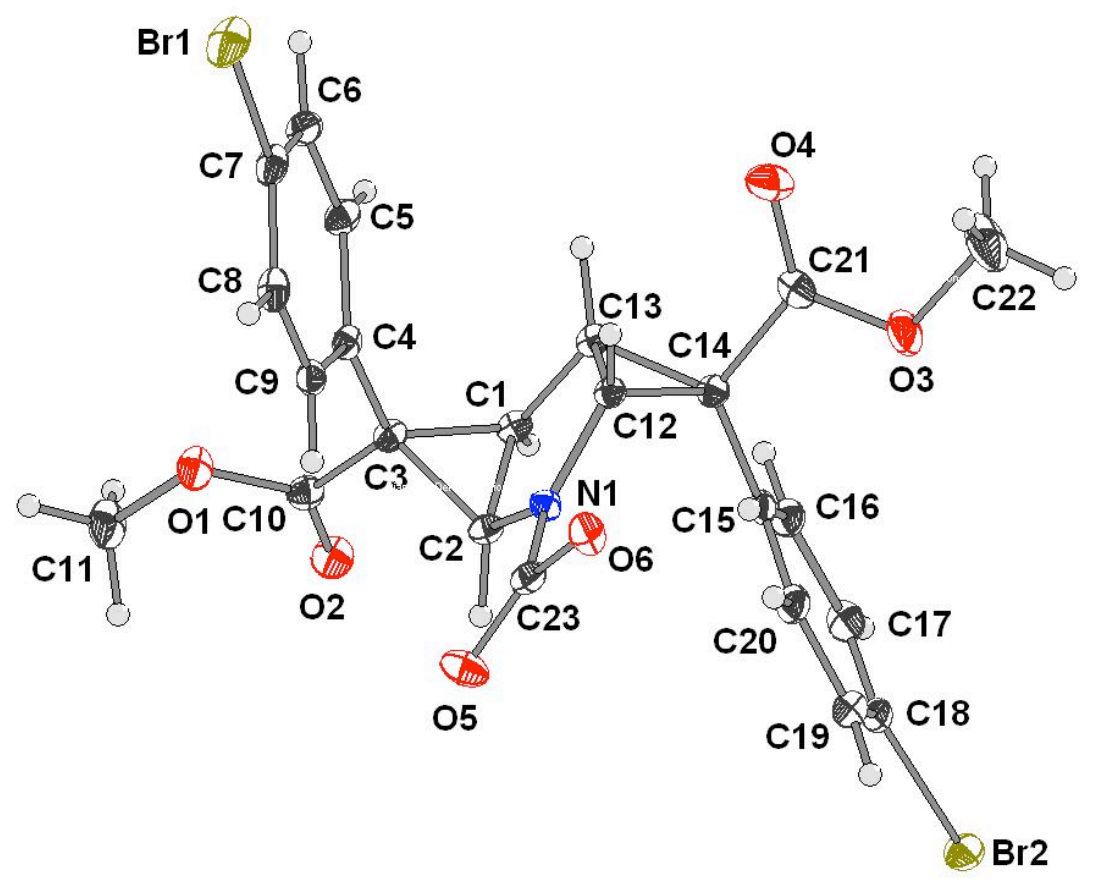

Fig.1. ORTEP plot and label scheme for 5. Displacement ellipsoids are drawn at the 50\% probability level. tert-Butyl group bonded to $\mathrm{O}(6)$ is omitted for clarity.

This compound was recrystallized by dissolving in diethyl ether, and then placed in a larger sealed vessel containing pentane, which diffused into solution to give colorless prisms. 


\section{X-ray Crystal Structure of 9}

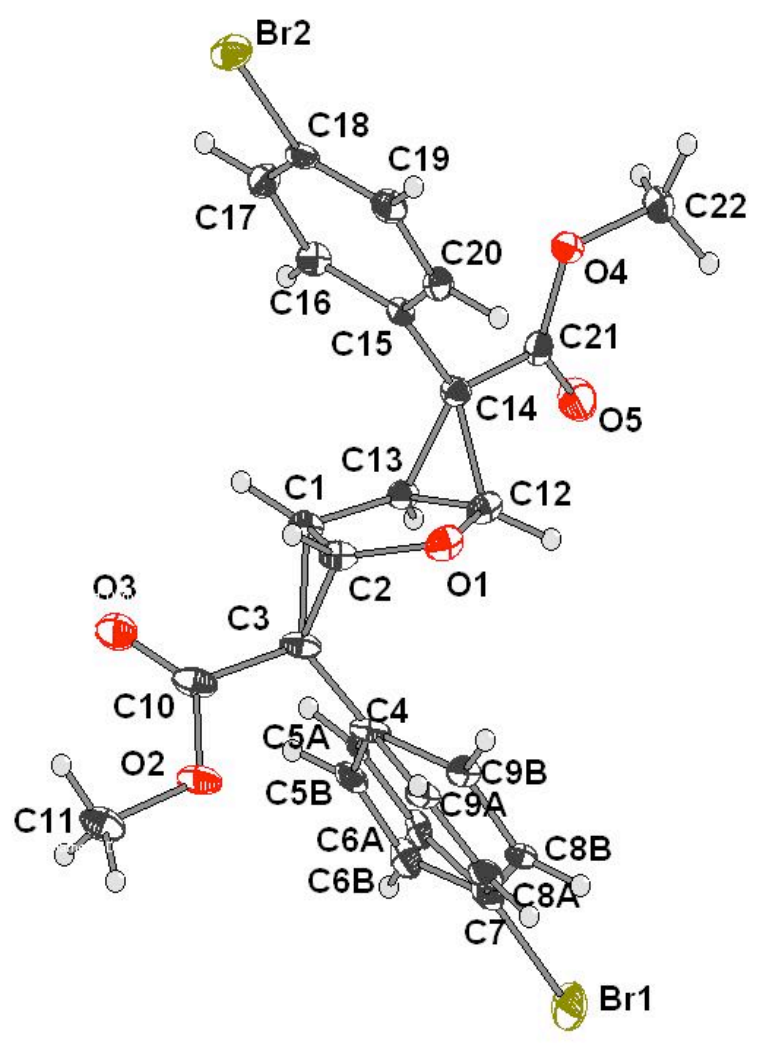

Fig.2. ORTEP plot and label scheme for 9. Displacement ellipsoids are drawn at the 50\% probability level.

This compound was recrystallized by dissolving in diethyl ether, and then placed in a larger sealed vessel containing pentane, which diffused into solution to give colorless plates. 


\section{X-ray Crystal Structure of ent-12}

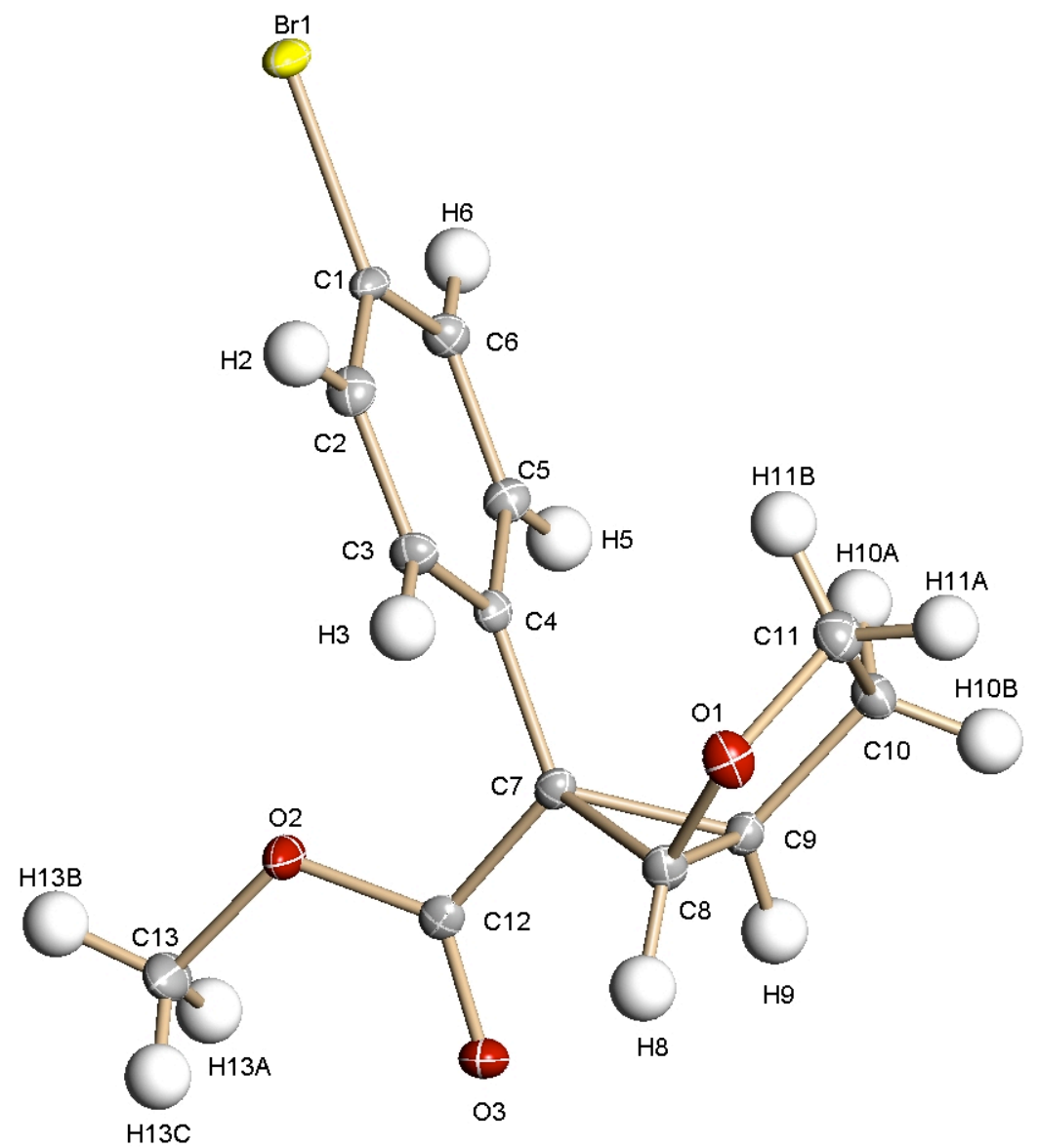

Fig.3. ORTEP plot and label scheme for ent-12. Displacement ellipsoids are drawn at the $50 \%$ probability level.

This compound was recrystallized by dissolving in diethyl ether, and then placed in a larger sealed vessel containing pentane, which diffused into solution to give colorless blocks. 


\section{X-ray Crystal Structure of 15}

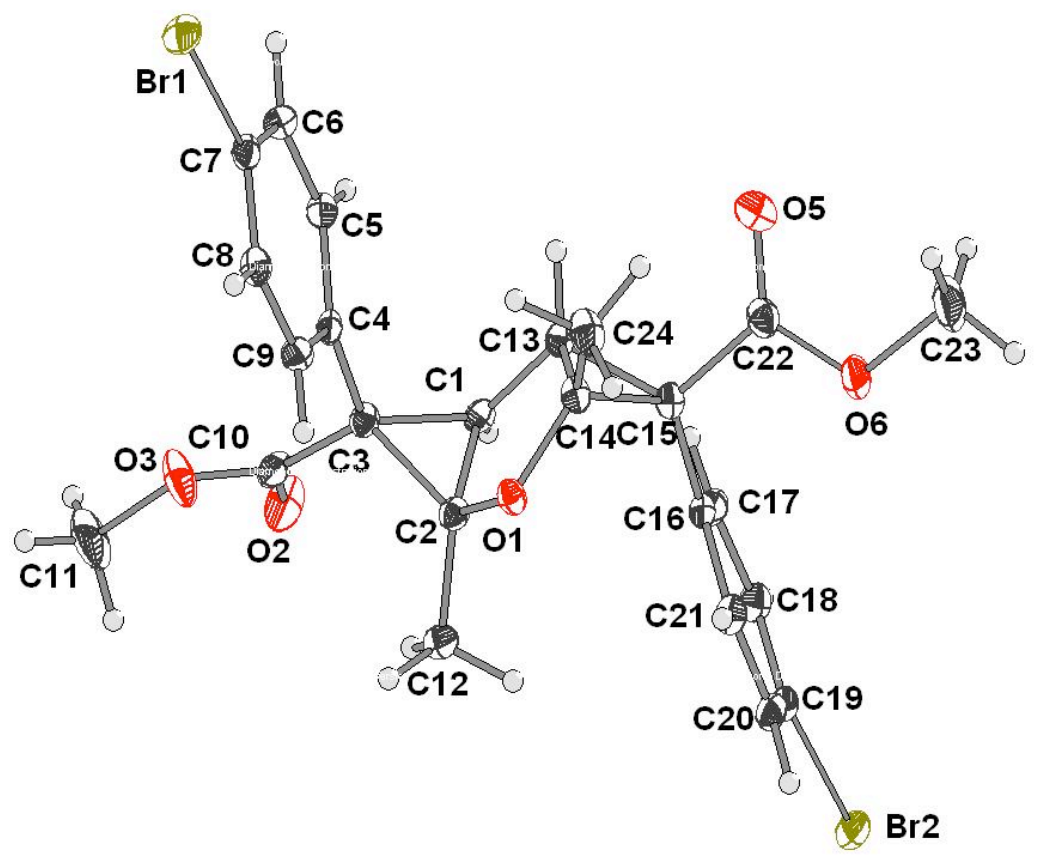

Fig.4. ORTEP plot and label scheme for 15. Displacement ellipsoids are drawn at the $50 \%$ probability level.

This compound was recrystallized by dissolving in diethyl ether, and then placed in a larger sealed vessel containing pentane, which diffused into solution to give colorless blocks. 


\section{X-ray Crystal Structure of 20}

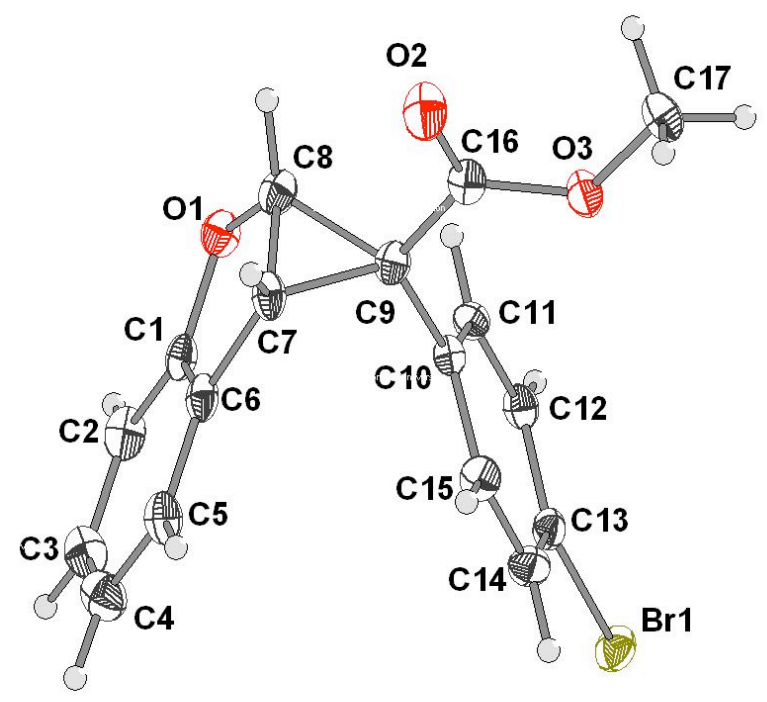

Fig.5. ORTEP plot and label scheme for 1. Displacement ellipsoids are drawn at the 50\% probability level.

This compound was recrystallized by dissolving in hot hexane, and then allowing to cool to room temperature. Slow evaporation of the solvent gave colorless prisms. 


\section{X-ray Crystal Structure of 32}

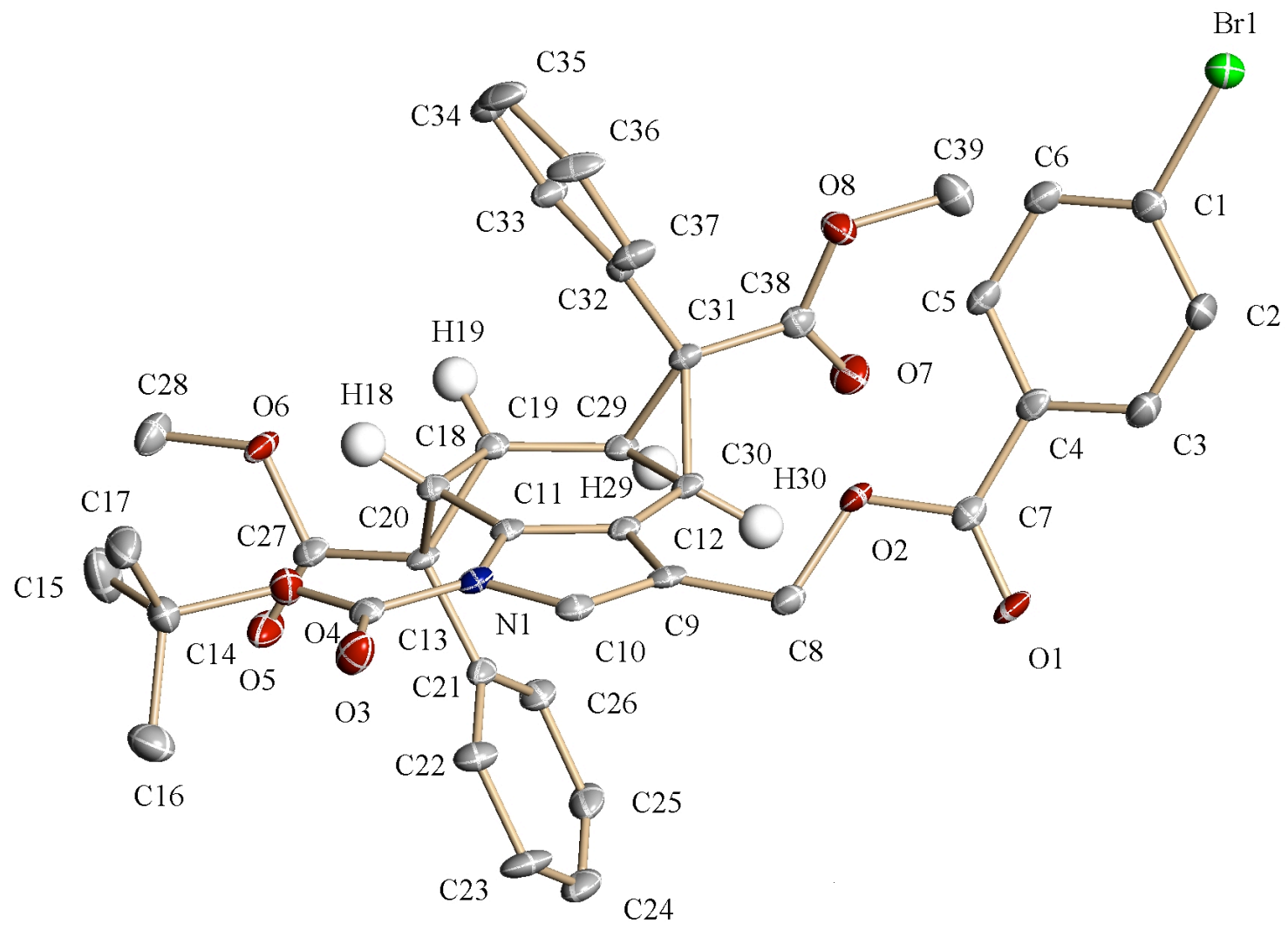

Figure 6. ORTEP plot and label scheme for 32. Displacement ellipsoids are drawn at the $50 \%$ probability level. Most hydrogen atoms are omitted for clarity.

This compound was recrystallized by dissolving in hot ethanol, and then allowing to cool to room temperature. Slow evaporation of the solvent gave colorless blocks. 


\section{Circular Dichroism Spectra for 20}
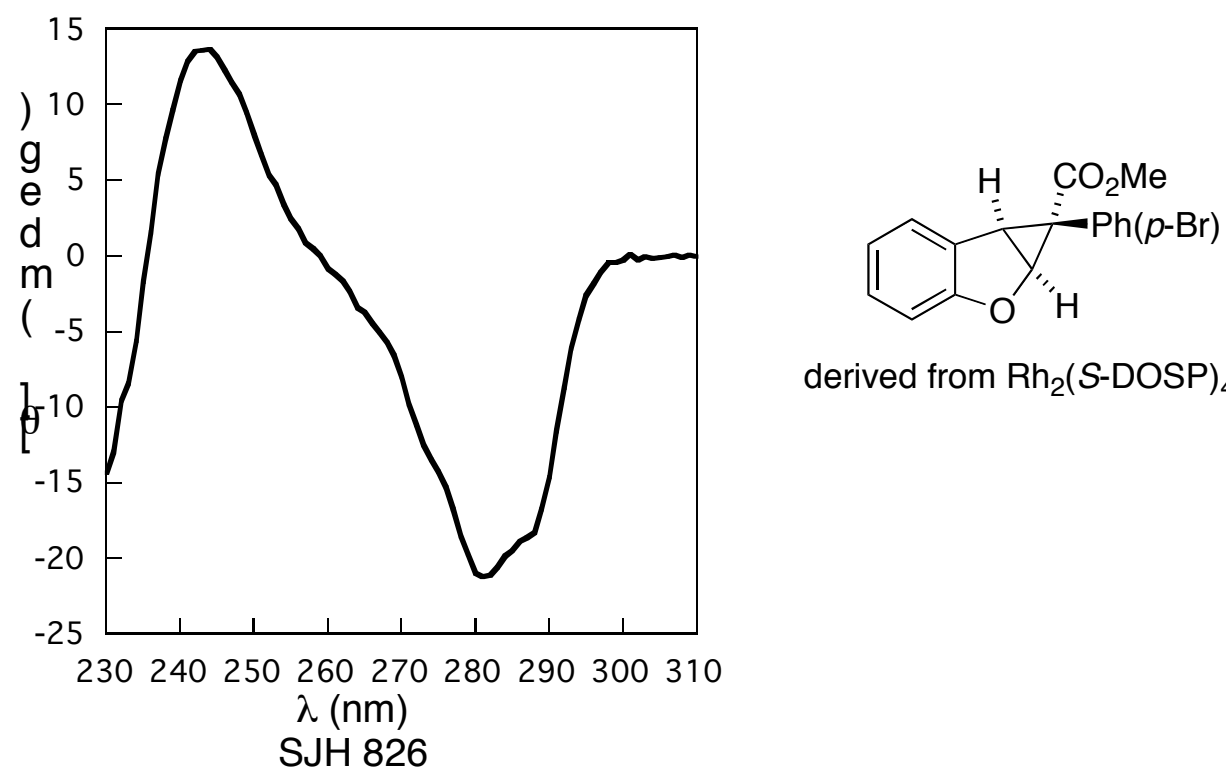

derived from $\mathrm{Rh}_{2}(S \text {-DOSP })_{4}$
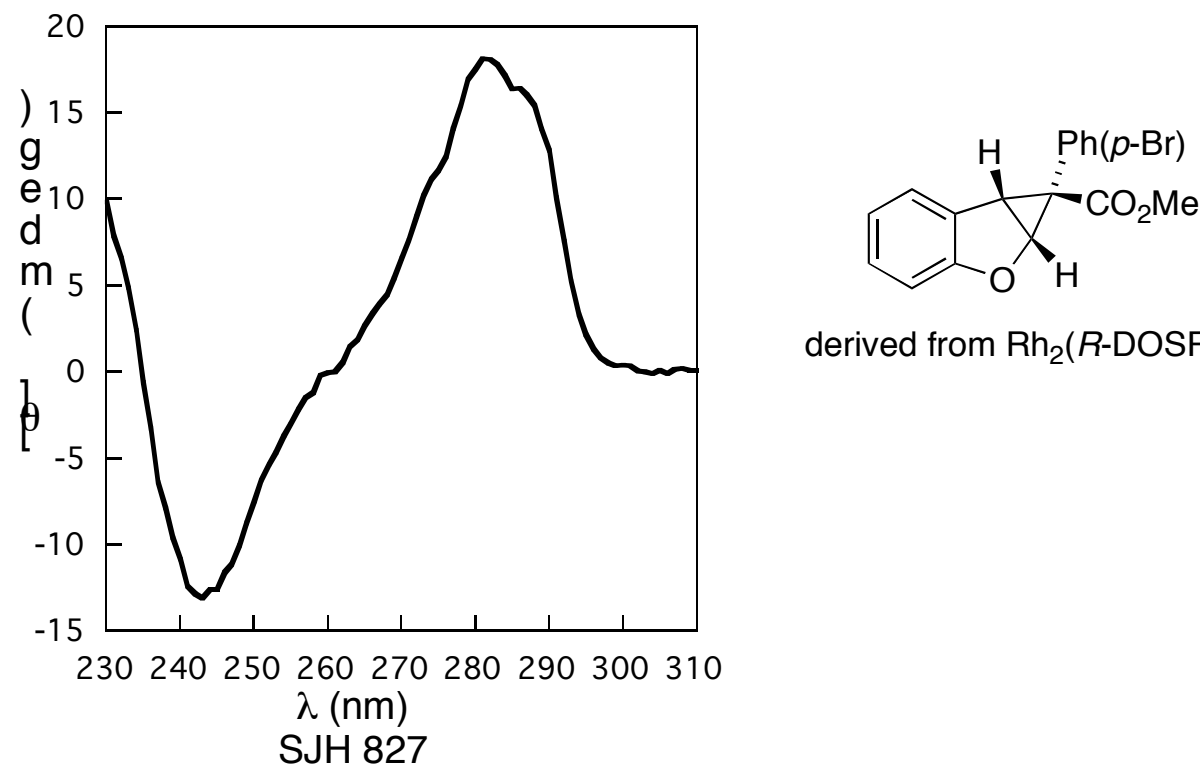

derived from $\mathrm{Rh}_{2}(R \text {-DOSP })_{4}$ 


\section{Circular Dichroism Spectra for 21}
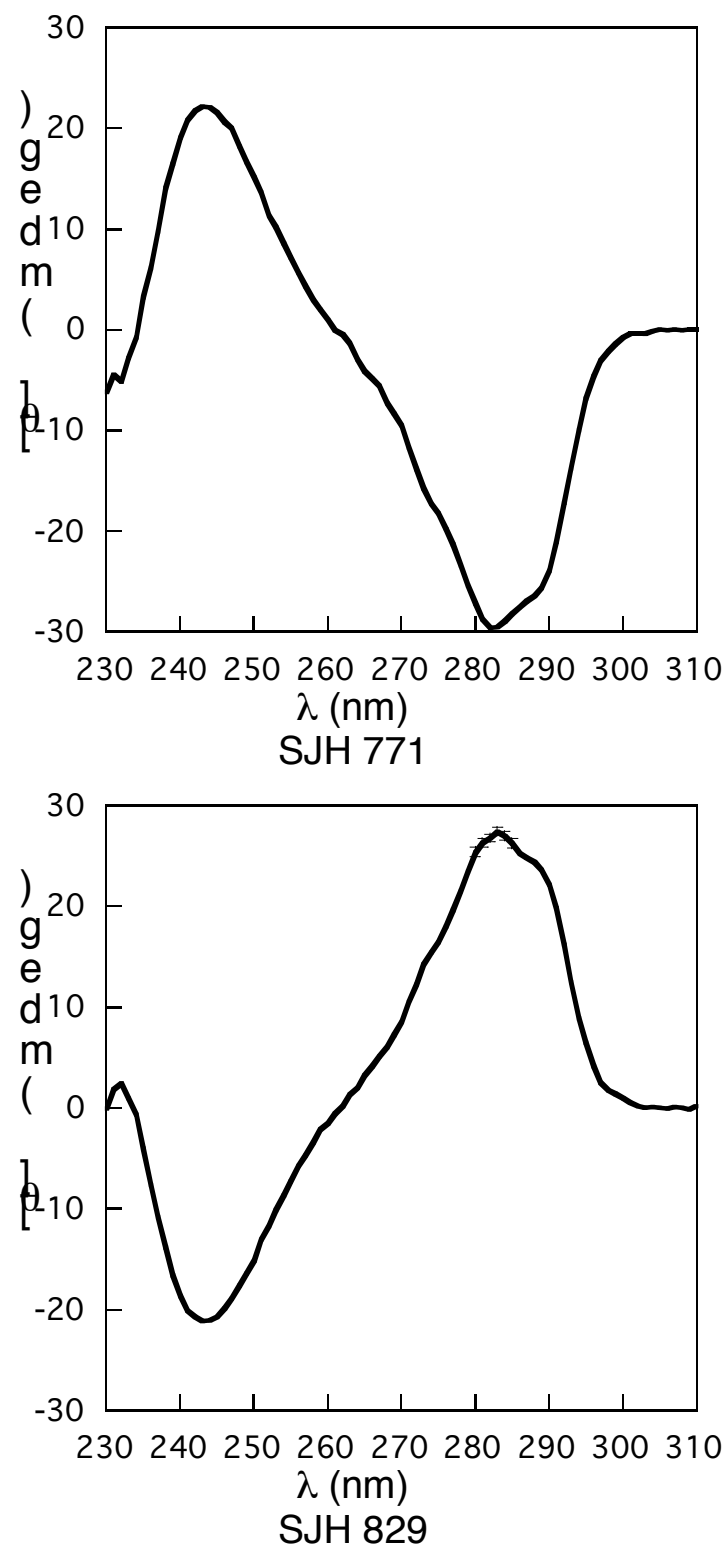

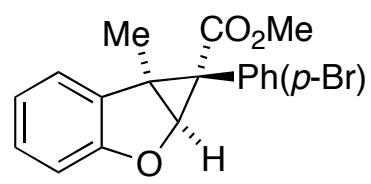

derived from $\mathrm{Rh}_{2}(S \text {-DOSP })_{4}$

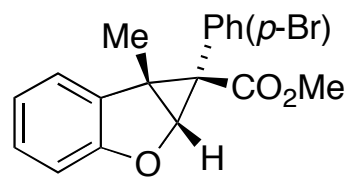

derived from $\mathrm{Rh}_{2}(R \text {-DOSP })_{4}$ 